This is the accepted manuscript made available via CHORUS. The article has been published as:

Method for separating jets and the underlying event in heavy ion collisions at the BNL Relativistic Heavy Ion Collider

J. A. Hanks, A. M. Sickles, B. A. Cole, A. Franz, M. P. McCumber, D. P. Morrison, J. L. Nagle, C. H. Pinkenburg, B. Sahlmueller, P. Steinberg, M. von Steinkirch, and M. Stone Phys. Rev. C 86, 024908 - Published 10 August 2012 DOI: 10.1103/PhysRevC.86.024908 


\title{
Method for Separating Jets and the Underlying Event in Heavy Ion Collisions at the Relativistic Heavy Ion Collider
}

\author{
J. A. Hanks ${ }^{1}$, A. M. Sickles², B. A. Cole ${ }^{3}$, A. Franz ${ }^{2}$, M. P. McCumber ${ }^{4}$, D. P. Morrison², \\ J. L. Nagle ${ }^{4}$, C. H. Pinkenburg ${ }^{2}$, B. Sahlmueller ${ }^{1}$, P. Steinberg ${ }^{2}$, M. von Steinkirch ${ }^{1}$, M. Stone ${ }^{4}$ \\ 1 Department of Physics and Astronomy, Stony Brook University, \\ SUNY, Stony Brook, New York 11794-3400, USA \\ 2 Physics Department, Brookhaven National Laboratory, Upton, New York, 11973-5000 \\ ${ }^{3}$ Columbia University, New York, New York 10027 and Nevis Laboratories, Irvington, New York 10533, USA and \\ 4 University of Colorado, Boulder, Colorado 80309, USA
}

(Dated: July 6, 2012)

\begin{abstract}
Reconstructed jets in heavy ion collisions are a crucial tool for understanding the quark-gluon plasma. The separation of jets from the underlying event is necessary particularly in central heavy ion reactions in order to quantify medium modifications of the parton shower and the response of the surrounding medium itself. There have been many methods proposed and implemented for studying the underlying event substructure in proton-proton and heavy ion collisions. In this paper, we detail a method for understanding underlying event contributions in $\mathrm{Au}+\mathrm{Au}$ collisions at $\sqrt{s_{N N}}=200$ GeV utilizing the HIJING event generator [1]. This method, extended from previous work by the ATLAS collaboration [2], provides a well-defined association of "truth jets" from the fragmentation of hard partons with "reconstructed jets" using the anti- $k_{T}$ algorithm. Results presented here are based on an analysis of $750 \mathrm{M}$ minimum bias HIJING events. We find that there is a substantial range of jet energies and radius parameters where jets are well separated from the background fluctuations (often termed "fake jets") that make jet measurements at RHIC a compelling physics program.
\end{abstract}

\section{INTRODUCTION}

Understanding the detailed interaction and coupling between hard scattered partons and the quark-gluon plasma through which they propagate is essential to our fundamental knowledge of QCD and in determining properties of the quark-gluon plasma. The measurement of fully reconstructed jets in heavy ion collisions at the LHC [3, 4] highlight the substantial additional information contained therein and its complementary nature to single hadron [5-7], di-hadron correlations [8-11]. The measurement of direct photon-jet correlations is another critical handle to be utilized [12]. Extending fully calorimetric jet measurements to lower center-of-mass energies at the Relativistic Heavy Ion Collider provides measurements for kinematics difficult to access at the LHC and the QGP at different temperature and coupling regime.

With the first $\mathrm{Pb}+\mathrm{Pb}$ at $\sqrt{s_{N N}}=2.76 \mathrm{TeV}$ collisions at the LHC new insights into jet physics in heavy ion collisions were gained. The ATLAS collaboration reported an increase in the number of energy asymmetric di-jets in central $\mathrm{Pb}+\mathrm{Pb}$ collisions compared to proton-proton and peripheral $\mathrm{Pb}+\mathrm{Pb}$ collisions [3]. They also reported the suppression of jets with $100<p_{T}<200 \mathrm{GeV} / \mathrm{c}$ by a factor of approximately two when comparing central to peripheral $\mathrm{Pb}+\mathrm{Pb}$ collisions [13]. The CMS collaboration measured jet-hadron correlations in a similar jet $p_{T}$ range and found that the energy lost by high $p_{T}$ fragments was approximately balanced by very low $p_{T}$ tracks far from the jet axis [4]. However the data from both RHIC and the initial LHC results are not enough to constrain the physics of jet quenching. Most theoretical descriptions have relied on weakly coupled techniques [14]. Features of strong coupling, as observed in descriptions of the bulk matter, might contribute to jet quenching as well. More data on jet observables (including dijet, $\gamma$-jet and heavy flavor tagged jets) at RHIC and the LHC will be necessary to understand the physics of jet quenching over the full range of medium properties and jet kinematics and probe for sensitivity of the quenching to outgoing parton virtuality.

The multiplicity of charged particles $d N_{c h} / d \eta$ is approximately 2.15 times higher for $\mathrm{Pb}+\mathrm{Pb}$ central collisions at $\sqrt{s_{N N}}=2.76 \mathrm{TeV}$ compared with $\mathrm{Au}+\mathrm{Au}$ central collisions at $\sqrt{s_{N N}}=200 \mathrm{GeV}$ [15]. Thus the soft particle background is substantially higher for LHC events. However, the jet cross section is substantially higher as well, and measurements for jets with energies greater than $100 \mathrm{GeV}$ appear well separated from the background (though detailed publications of these studies are not yet available). Various methods have been explored at the LHC and RHIC for understanding the underlying event contributions, and background fluctuations reconstructed as jets, so called "fake jets" [2, 16-19].

At $\sqrt{s_{N N}}=200 \mathrm{GeV}$ the projected jet rates into $|\eta|<1$ based on NLO pQCD cross sections [20] and expected RHIC luminosities have been computed [21]. In a typical year of RHIC running 50B Au+Au events could be sampled. In the top $20 \%$ centrality that would lead to approximately $10^{7}$ jets above $20 \mathrm{GeV}, 10^{6}$ jets above 30 $\mathrm{GeV}, 10^{5}$ jets above $40 \mathrm{GeV}$ and $10^{4}$ jets above $50 \mathrm{GeV}$. Over $60 \%$ of the time there is full containment of the opposing dijet for $20 \mathrm{GeV}$ jets within $|\eta|<1$ with that percentage increasing with increasing jet energy.

In this paper, we present a study of jet reconstruction and separation from the underlying event using 
the HIJING [1] event generator for $\mathrm{Au}+\mathrm{Au}$ events at $\sqrt{s_{N N}}=200 \mathrm{GeV}$. This follows an iterative underlying event subtraction procedure extended from one developed by the ATLAS Collaboration [2]. While the exact definition of a correctly reconstructed jet versus a "fake jet" is arbitrary, this methodology allows us to make a well-defined and documented comparison to cross-check with other methods. These studies were inspired by the proposed sPHENIX upgrade to the PHENIX detector [21], however they apply to any calorimeter based jet measurements at $\sqrt{s_{N N}}=200 \mathrm{GeV}$.

\section{JET - UNDERLYING EVENT SEPARATION METHODOLOGY}

For these studies we utilize the HIJING (version 1.383) event generator run with standard settings and quenching turned off for $\mathrm{Au}+\mathrm{Au}$ collisions at $\sqrt{s_{N N}}=200 \mathrm{GeV}$. HIJING is a QCD based Monte Carlo for the study of jet production in high energy nucleus-nucleus collisions. For these initial studies, we explore what a perfect resolution detector is capable of measuring. We assume a segmentation in $\Delta \eta \times \Delta \phi=0.1 \times 0.1$ and that all particle energies are recorded perfectly (with the exception of neutrinos and muons). We assume a nominal coverage of $|\eta|<1.0$ and full azimuthal coverage. For the entire study we utilize the anti- $k_{T}$ jet reconstruction algorithm [22] (part of the FastJet package [23]) with radius parameters $\mathrm{R}=0.2$, 0.3 , and 0.4 .

A schematic diagram of the underlying event subtraction steps is shown in Fig. 1. The first step is to run the anti- $k_{T}$ algorithm over the full set of energy values (unsubtracted) with $\mathrm{R}=0.2$ and record the jet axis coordinates in $\eta$ and $\phi$. This initial suite of jet candidates is used to exclude regions around these jets from the initial underlying event average energy. Exclusion regions are defined by $R=0.2$ jets in which the maximum tower in the jet has an energy of more than three times the average tower energy in the jet. We then exclude all energies for $0.1 \times 0.1$ cells whose center coordinate is within $\Delta R=\sqrt{\Delta \eta^{2}+\Delta \phi^{2}}<0.4$ of any of the above initial jet candidates. The remaining energy values are used to determine the average cell energy (i.e. in the nonjet regions) in $\Delta \eta=0.1$ strips. The modulation in the background due to flow must first be removed, so the $\langle\cos (2 \phi)\rangle$ (i.e. $v_{2}$ parameter) is also determined for the energy distribution and removed from each cell before determining the average. Only the $\eta$ strips which have complete $\phi$ coverage after the determination of the exclusion regions are used in the $v_{2}$ determination.

The HIJING generator has no bulk collective flow and thus has only a modest $\langle\cos (2 \phi)\rangle$ from decays, di-jet correlations and global momentum conservation. As the flow modulation of the underlying event is an important component of any subtraction procedure on real data, we have added a flow modulation to the individual HIJING particles prior to segmenting the energies into cells. The flow parameterization [24] is based on fits to the available data. Higher flow moments have an increasing relative importance for more central events [25], and can be incorporated in future studies.

This underlying event average energy is a zeroth order estimate since the initial jet determination does not have an underlying event subtraction. We now subtract the $v_{2}$ modulated underlying event energy cell-by-cell from the cells contained by the initial set of $R=0.2$ jets to get a better estimate of the jet $E_{T}$. At this second iterative step, new exclusion regions are defined by towers with $\Delta R<0.4$ around background subtracted jets with $E_{T}>$ $20 \mathrm{GeV}$. The underlying event and $v_{2}$ are re-determined removing towers within $\Delta \eta<0.4$ of the jets as described above and the background re-subtracted from the original unsubtracted towers. Finally the anti- $k_{T}$ jet algorithm is run on the background subtracted towers with a range of $\mathrm{R}$ values $(0.2,0.3,0.4)$.

When the jet reconstruction is run over background subtracted towers many of the towers have negative $E_{T}$. We modify these towers to have a small positive energy before passing them to the jet reconstruction algorithm. After the towers are grouped into jets we recalculate the jet $E_{T}$ including the negative energy.

\section{HIJING TRUTH INFORMATION}

In order to identify "true jets" from the HIJING event generator, we have augmented the code so that every time the fragmentation routine (HIJFRG) is called, we record the set of final state hadrons that result from that fragmentation. We then run the anti- $k_{T}$ algorithm on those final state hadrons (using their exact momentum vectors). The jet reconstruction is run once for each anti$k_{T}$ jet $\mathrm{R}$ parameter under consideration and the resulting "true jet" information is recorded.

Before presenting the results, it is important to define our terms. Even in a model such a HIJING where all truth information is known, there is an arbitrariness in the definitions of "fake jets" and "true jets" as examples. For example, consider a HIJING fragmentation call that results in hadrons reconstructed via anti- $k_{T}$ with $\mathrm{R}=$ 0.4 into a jet with an energy of $20 \mathrm{GeV}$. If after running jet reconstruction over the full HIJING event one reconstructs a jet using anti- $k_{T}$ with $\mathrm{R}=0.4$ that has a jet axis within $\Delta R \equiv \sqrt{\Delta \eta^{2}+\Delta \phi^{2}}<0.25$ and energy 18 $\mathrm{GeV}$, this is clearly a true jet and the $2 \mathrm{GeV}$ difference is a result of the fluctuations in the underlying event. On the other hand, the case of a true HIJING jet with an energy of $4 \mathrm{GeV}$ and being reconstructed in the midst of the underlying event with an energy of $40 \mathrm{GeV}$ is clearly a fake jet (i.e. a very small jet that combined with substantial background fluctuations that results in a very large energy reconstruction).

A jet is a good "true jet" if there is an associated HIJING fragmentation jet within $\Delta R=\sqrt{\Delta \eta^{2}+\Delta \phi^{2}}<$ 0.25 and greater than $5 \mathrm{GeV}$. Here, we define a "fake 
FIG. 1: Schematic illustration of the jet background subtraction method.

FIG. 2: Event display for a $E_{T}=18 \mathrm{GeV}$ true dijet pair jet matched to a $E_{T}=15$ and $16 \mathrm{GeV}$ reconstructed jets in $\mathrm{a} b=1.8 \mathrm{fm}$ HIJING event. All jets shown in this event display are reconstructed with the anti- $k_{T}$ algorithm with $\mathrm{R}=0.2$. Both histograms show the background subtracted $0.1 x 0.1 \eta-\phi$ tower energy. A minimum $E_{T}$ cut of $5 \mathrm{GeV}$ is placed on all jets shown in this display. The stars in the left panel show the true HIJING jets and box below shows jet $E_{T}$ and $\eta, \phi$ location of the jet axis. The right panel shows the reconstructed jets. The jet labeled R1 is reconstructed at $E_{T}=16 \mathrm{GeV}$ and matched to the $\mathrm{H} 1$ jet in the left panel and $\mathrm{R} 2$ is matched to $\mathrm{H} 2$. The other reconstructed jet in the event with $E_{T}>5 \mathrm{GeV}$ is shown as R3. It is not associated with any true jets with $E_{T}>5 \mathrm{GeV}$.

jet" as one where the associated HIJING fragmentation jet is less than $5 \mathrm{GeV}$ (or does not exist at all). We then examine in detail the HIJING fragmentation jet energy distribution for those associated with different selected fully reconstructed jet energies. In principle, one could introduce no such arbitrary definition and put everything into a response matrix down to the lowest energy scales. In practice, if there are substantial contributions of very low energy HIJING fragmentation jet energies to high energy reconstructed jets it will be nearly impossible to control the systematics and unfold such a matrix.

Results presented here are based on an analysis of 750M minimum bias HIJING events.

\section{RESULTS}

In order to illustrate the background subtraction procedure, we show a selection of event displays. Figure 2 shows a true dijet pair with $\mathrm{R}=0.2$ where both jets have been matched to reconstructed jets. The reconstructed jet has an axis within $\Delta R<0.1$ of the true. Also shown in the event are the next highest $E_{T}$ reconstructed jet. This jet is not matched to any true jets with $E_{T}>5 \mathrm{GeV}$ and has $E_{T}$ in the region where we expect fake jets to dominate. Figure 3 shows a fake jet with $E_{T}=30 \mathrm{GeV}$ which is not matched to any true jet from the HIJING event. One other fake jet, also not matched to any true jets, is shown on the plot.

We concentrate on central collisions where the underlying event background is largest. For this study we define collision centrality in the HIJING events by the number of charged particles with psuedorapidity $3<\eta<4$. Figure 4 shows the efficiency of finding matches to true jets in the most central $10 \%$ of collisions for the various anti- $k_{T} \mathrm{R}$ parameters as a function of the true jet $E_{T}$.
FIG. 3: Event display for a $E_{T}=30 \mathrm{GeV}$ fake jet. All jets shown in this event display are reconstructed with the anti- $k_{T}$ algorithm with $\mathrm{R}=0.4$. Both histograms show the background subtracted $0.1 \times 0.1 \eta-\phi$ tower energy. A minimum $E_{T}$ cut of $5 \mathrm{GeV}$ is placed on all jets shown in this display. There are no true HIJING jets with $E_{T}>5 \mathrm{GeV}$ in this event. The right panel shows the reconstructed jets. The jet labeled R1 is reconstructed at $E_{T}=30 \mathrm{GeV}$. The other reconstructed jet in the event with $E_{T}>5 \mathrm{GeV}$ is shown as R2. The jet $E_{T}$ and $\eta, \phi$ locations are shown in the bottom right box.

FIG. 4: Matching efficiency for true HIJING jets as a function of jet $E_{T}$ for anti- $k_{T}$ R parameters 0.2 (black), 0.3 (red) and 0.4 (blue). For a jet to be considered matched the reconstructed jet axis must be within $\Delta R<0.25$ of the HIJING jet axis.

For all $\mathrm{R}$ parameters the efficiency rises with jet $E_{T}$ and approaches $100 \%$ between 20 and $30 \mathrm{GeV}$.

However, in order to quantify the jet performance we need to understand the contribution to the reconstructed jet $E_{T}$ spectrum from jets which are not matched to any true HIJING jet, "fake jets". In Figure 5 we show the true, reconstructed and fake jet $E_{T}$ spectra for $\mathrm{R}=0.2$ (left), 0.3 (middle) and 0.4 (right) for the $10 \%$ most central $\mathrm{Au}+\mathrm{Au}$ at $\sqrt{s_{N N}}=200 \mathrm{GeV}$ HIJING events. Shown as red are the true HIJING fragmentation jet distributions. The points show the final reconstructed jet distribution. This is broken down into those jets that are matched with a true HIJING jet and those that are not matched with a true HIJING jet. To be considered matched the jet axis of the reconstructed jet must be within $\Delta R<0.25$ of the true HIJING jet and the HIJING jet must have $E_{T}>5 \mathrm{GeV}$. One observes a good match between true HIJING and matched reconstructed jet distributions taking into account the additional energy resolution blurring from the underlying event subtraction. One observes a very large fraction of reconstructed jets are not matched at low $E_{T}$; the fraction then falls quickly and goes below the matched reconstructed jets at around $18 \mathrm{GeV}$ in the $\mathrm{R}=0.2$ case. The crossing point is at higher $E_{T}$ for $\mathrm{R}=0.3$ and 0.4 jets, around 25 and $30 \mathrm{GeV}$, respectively.

The low fake rate alone is not sufficient to claim the jet reconstruction is well under control due to the arbitrariness of the $5 \mathrm{GeV}$ separation between "fake" and "true" jet fragmentation associations. Shown in Figure 6 is the distribution of HIJING true energies for fully reconstructed jet energies (with different selections). The 
FIG. 5: $E_{T}$ spectra for true HIJING jets (red line) and reconstructed jets (black points). The reconstructed jets are further divided into those which are matched to a true HIJING jet (blue line) and those which are not matched to a true HIJING jet ("fake jets", black line). To be considered matched the axis of the true HIJING jet and the reconstructed jet must be within $\Delta R<0.25$ and the HIJING jet must have $E_{T}>5 \mathrm{GeV}$. Shown are results for $0-10 \%$ central HIJING events using anti- $k_{T}$ jets with $\mathrm{R}=0.2(\mathrm{a}), \mathrm{R}=0.3$ (b) and $\mathrm{R}=0.4$ (c).

upper left panel for reconstructed jets with $\mathrm{R}=0.2$ and energies $15-20 \mathrm{GeV}$ shows a peaked distribution around $\approx 15 \mathrm{GeV}$. The tail to lower energies could in principle be accounted for in a response matrix (though with great care and systematic cross checks). However, as one moves to higher energies $25-30 \mathrm{GeV}$, there is a peak around $26 \mathrm{GeV}$ with a width of $5 \mathrm{GeV}$ and essentially no tail contribution under the peak. This indicates a regime where a standard response matrix and unfolding procedure should be successful. Similar plots are shown for R $=0.3$ and $\mathrm{R}=0.4$. There is a shift downward from the reconstructed jet energies to the corresponding true jet energies due to the rapid fall off of the jet cross section with energy and a tail to low HIJING jet energies that disappears with increasing reconstructed jet $E_{T}$ and the corresponding decrease of fake jets.

In order to quantify the purity of the reconstructed jet sample, we have fit the distributions with a background contribution which falls exponentially with increasing jet $E_{T}$ and a Gaussian with a free mean and width. The results of those fits, along with the fractions of the total reconstructed jets (both matched and unmatched) which are included in the Gaussian are shown in Table I.

In addition to the fake jet contribution to the reconstructed jet sample it is also important to quantify the jet energy resolution and scale for our algorithm. In order to do this we have embedded PYTHIA [26] (version 6.421) jets into our HIJING events. One PYTHIA event with a high $p_{T}$ dijet was embedded into every HIJING event. The PYTHIA and reconstructed jets are required to obey the same matching cut of $\Delta R<0.25$ as the fake jet study discussed above. The jet energy resolution and jet energy scale are shown in Figure 9 the anti- $k_{T} \mathrm{R}$ parameters 0.2 and 0.4 for central HIJING events and PYTHIA events (not embedded into HIJING) put into towers in the same manner as the HIJING events. The jet energy resolution improves with increasing jet energy and decreasing jet $\mathrm{R}$ as expected. The energy scale, $\frac{\left\langle E_{T, \text { reco }}-E_{T, \text { true }}\right\rangle}{E_{T, \text { true }}}$ is within $\approx 5 \%$ of zero for the anti- $k_{T}$ R parameters considered here. The energy offset for the PYTHIA jets is due to the imposed tower segmentation. For the purposes of this study we did not pursue further refinements. A similar resolution evaluation has been done by the CMS collaboration [4].

\section{CONCLUSIONS}

We have performed a HIJING study of jet reconstruction using an iterative background subtraction method

\begin{tabular}{|c|c|c|c|}
\hline \multicolumn{4}{|c|}{$\mathrm{R}=0.2$} \\
\hline$\left\langle E_{T, \text { reco }}>(\mathrm{GeV})\right.$ & $\left\langle E_{T, \text { true }}>(\mathrm{GeV})\right.$ & $\sigma_{E_{T}}$ & $\frac{S}{S+B}$ \\
\hline $22.1 \pm 0.00406$ & $14.8 \pm 0.0746$ & $5.7 \pm 0.0374$ & 0.164 \\
\hline $27.2 \pm 0.00909$ & $20 \pm 0.0865$ & $5.63 \pm 0.0825$ & 0.522 \\
\hline $32.2 \pm 0.0185$ & $24.2 \pm 0.114$ & $5.66 \pm 0.11$ & 0.73 \\
\hline $37.5 \pm 0.0423$ & $29.3 \pm 0.254$ & $6.66 \pm 0.195$ & 0.87 \\
\hline \hline \multicolumn{4}{|c|}{$\mathrm{R}=0.3$} \\
\hline$<E_{T, \text { reco }}>(\mathrm{GeV})$ & $<E_{T, \text { true }}>(\mathrm{GeV})$ & $\sigma_{E_{T}}$ & $\frac{S}{S+B}$ \\
\hline $22.1 \pm 0.00748$ & $14.9 \pm 0.124$ & $5.59 \pm 0.0634$ & 0.174 \\
\hline $27.2 \pm 0.0169$ & $19.7 \pm 0.179$ & $5.83 \pm 0.144$ & 0.576 \\
\hline $32.2 \pm 0.0354$ & $23.7 \pm 0.261$ & $6.33 \pm 0.211$ & 0.802 \\
\hline $37.5 \pm 0.072$ & $29.6 \pm 0.329$ & $7.04 \pm 0.306$ & 0.908 \\
\hline \hline \multicolumn{4}{|c|}{$\mathrm{R}=0.4$} \\
\hline$<E_{T, \text { reco }}>(\mathrm{GeV})$ & $<E_{T, \text { true }}>(\mathrm{GeV})$ & $\sigma_{E_{T}}$ & $\frac{S}{S+B}$ \\
\hline $27.1 \pm 0.00543$ & $14.2 \pm 0.159$ & $7.22 \pm 0.0749$ & 0.129 \\
\hline $32.2 \pm 0.0118$ & $20.8 \pm 0.255$ & $6.79 \pm 0.173$ & 0.358 \\
\hline $37.1 \pm 0.0232$ & $23.5 \pm 0.26$ & $7.39 \pm 0.183$ & 0.652 \\
\hline $42.5 \pm 0.0511$ & $27.9 \pm 0.478$ & $6.67 \pm 0.504$ & 0.512 \\
\hline
\end{tabular}

TABLE I: Jet parameters from fits to the plots in Figure 8 for $\mathrm{R}=0.4$ anti- $k_{T}$ jets with centrality from 0 - $10 \%$. $<E_{T, \text { reco }}>$ is the mean reconstructed jet $E_{T}$ within the $5 \mathrm{GeV}$ wide bins. $\left\langle E_{T, \text { true }}>\right.$ and $\sigma_{E T \text {, true }}$ are the mean and width from the Gaussian component in the fit and $\frac{S}{S+B}$ is the fraction of the area of the fit that is included in the Gaussian component rather than the exponential.

and electromagnetic and hadronic calorimetric information over $2 \pi$ in azimuth and $|\eta|<1$ with ideal response. We have shown that in this case we are able to reconstruct the input HIJING jets with a large signal to background with $E_{T}>20 \mathrm{GeV}$ for $\mathrm{R}=0.2$ jets, $30 \mathrm{GeV}$ for $\mathrm{R}=0.3$ jets and $40 \mathrm{GeV}$ for $\mathrm{R}=0.4$ jets. The results presented here are obtained without any additionally rejection of fake jets, though it is possible the reconstructed jet purities shown here could be further increased with fake jet rejection of some kind.

This study was designed to evaluate the feasibility of purely calorimetric jet measurements at RHIC. The results here are obtained using an ideal model of the detector and suggest promise for such measurements. This study is of course limited in scope. We have not taken into effect any detector effects aside from geometrical acceptance and granularity. More detailed studies need to be done to demonstrate the suitability of any particular detector design for jet measurements. 
FIG. 6: True $E_{T}$ for reconstructed jets anti- $k_{T} \mathrm{R}=0.2$ for the reconstructed jet $E_{T} 15-20 \mathrm{GeV}$ (a), 20-25 GeV (b), $25-30 \mathrm{GeV}$ (c) and 30-35 GeV (d). The lines show the results of fits containing a background component which is exponentially falling (dashed line) and a signal Gaussian component (dot-dashed line). The total fit is shown as a solid line. The plots show the $\frac{S}{S+B}$ where the signal (S) is determined from the area under the Gaussian within $\pm 2 \sigma$ of the mean and the total background (B) includes both those jets reconstructed with a $>2 \sigma$ energy mismatched and those which were not matched at all to a HIJING jet. Fit parameters are shown in Table I.

FIG. 7: True $E_{T}$ for reconstructed jets anti- $k_{T} \mathrm{R}=0.3$ for the reconstructed jet $E_{T} 20-25 \mathrm{GeV}$ (a), 25-30 GeV (b), 30-35 GeV (c), 35-40 GeV (d). The lines show the results of fits containing a background component which is exponentially falling (dashed line) and a signal Gaussian component (dot-dashed line). The total fit is shown as a solid line. The plots show the $\frac{S}{S+B}$ where the signal (S) is determined from the area under the Gaussian within $\pm 2 \sigma$ of the mean and the total background (B) includes both those jets reconstructed with a $>2 \sigma$ energy mismatched and those which were not matched at all to a HIJING jet. Fit parameters are shown in Table I.

\section{ACKNOWLEDGMENTS}

We would like to thank Yue Shi Lai for valuable discussions on the identification of jets in HIJING. Funding has been provided from the Department of Energy under contract number DE-AC02-98CH10886 (A.M.S, A.F, D.P.M, C.H.P, and P.S) and grant numbers DEFG02-96ER40988 (J.A.H, B.S.,M.vS) and DE-FG0203ER41244 (M.P.M, J.L.N, M.S).
[1] M. Gyulassy and X. Wang, Comput. Phys. Commun. 83, 307 (1994).

[2] ATLAS (ATLAS Collaboration) (2011), URL cdsweb.cern.ch/record/1353220/files/ ATLAS-CONF-2011-075.pdf.

[3] G. Aad et al. (Atlas Collaboration), Phys.Rev.Lett. 105, 252303 (2010).

[4] S. Chatrchyan et al. (CMS Collaboration), Phys.Rev. C84, 024906 (2011).

[5] K. Adcox et al. (PHENIX), Phys. Rev. Lett. 88, 022301 (2002).

[6] J. Adams et al. (STAR Collaboration), Phys.Rev.Lett. 91, 172302 (2003).

[7] A. Adare et al. (PHENIX), Phys. Rev. Lett. 101, 232301 (2008).

[8] J. Adams et al. (STAR Collaboration), Phys.Rev.Lett. 97, 162301 (2006).

[9] A. Adare et al. (PHENIX Collaboration), Phys.Rev. C78, 014901 (2008).

[10] B. Abelev et al. (STAR Collaboration), Phys.Rev. C80, 064912 (2009).

[11] A. Adare et al. (PHENIX), Phys. Rev. Lett. 104, 252301 (2010).

[12] X.-N. Wang, Z. Huang, and I. Sarcevic, Phys.Rev.Lett. 77, 231 (1996).
[13] P. Steinberg and A. Collaboration (2011), 1110.3352.

[14] A. Majumder and M. Van Leeuwen (2010), 1002.2206.

[15] K. Aamodt et al. (ALICE Collaboration), Phys.Rev.Lett. 106, 032301 (2011).

[16] Y. Lai (PHENIX) (2009), 0911.3399.

[17] P. Jacobs (STAR Collaboration) (2010), 1012.2406.

[18] M. Cacciari, G. P. Salam, and G. Soyez, Eur.Phys.J. C71, 1692 (2011).

[19] B. Abelev et al. (ALICE Collaboration) (2012), 1201.2423.

[20] W. Vogelsang, private communication.

[21] PHENIX (2010), URL \{http://www.phenix.bnl.gov/ phenix/WWW/docs/decadal/2010/phenix_decadal10_ full_refs.pdf\}.

[22] M. Cacciari, G. Salam, and G. Soyez, JHEP 0804, 063 (2008).

[23] M. Cacciari and G. P. Salam, Phys.Lett. B641, 57 (2006).

[24] M. Masera, G. Ortona, M. Poghosyan, and F. Prino, Phys. Rev. C79, 064909 (2009).

[25] A. Adare et al. (PHENIX Collaboration), Phys.Rev.Lett. 107, 252301 (2011).

[26] T. Sjostrand et al., Comput. Phys. Commun. 135, 238 (2001). 
FIG. 8: True $E_{T}$ for reconstructed jets anti- $k_{T} \mathrm{R}=0.4$ for the reconstructed jet $E_{T}$ 25-30 GeV (a), 30-35 GeV (b), $35-40 \mathrm{GeV}(\mathrm{c}), 40-45 \mathrm{GeV}$ (d). The lines show the results of fits containing a background component which is exponentially falling (dashed line) and a signal Gaussian component (dot-dashed line). The total fit is shown as a solid line. The plots show the $\frac{S}{S+B}$ where the signal $(\mathrm{S})$ is determined from the area under the Gaussian within $\pm 2 \sigma$ of the mean and the total background (B) includes both those jets reconstructed with a $>2 \sigma$ energy mismatched and those which were not matched at all to a HIJING jet. Fit parameters are shown in Table I.

FIG. 9: Jet energy resolution as a function of the true jet energy for jets reconstructed in 0-10\% central HIJING events. Shown in the plot are anti- $k_{T} \mathrm{R}=0.2$ (black), $\mathrm{R}=0.3$ (red) and $\mathrm{R}=0.4$ (blue). 


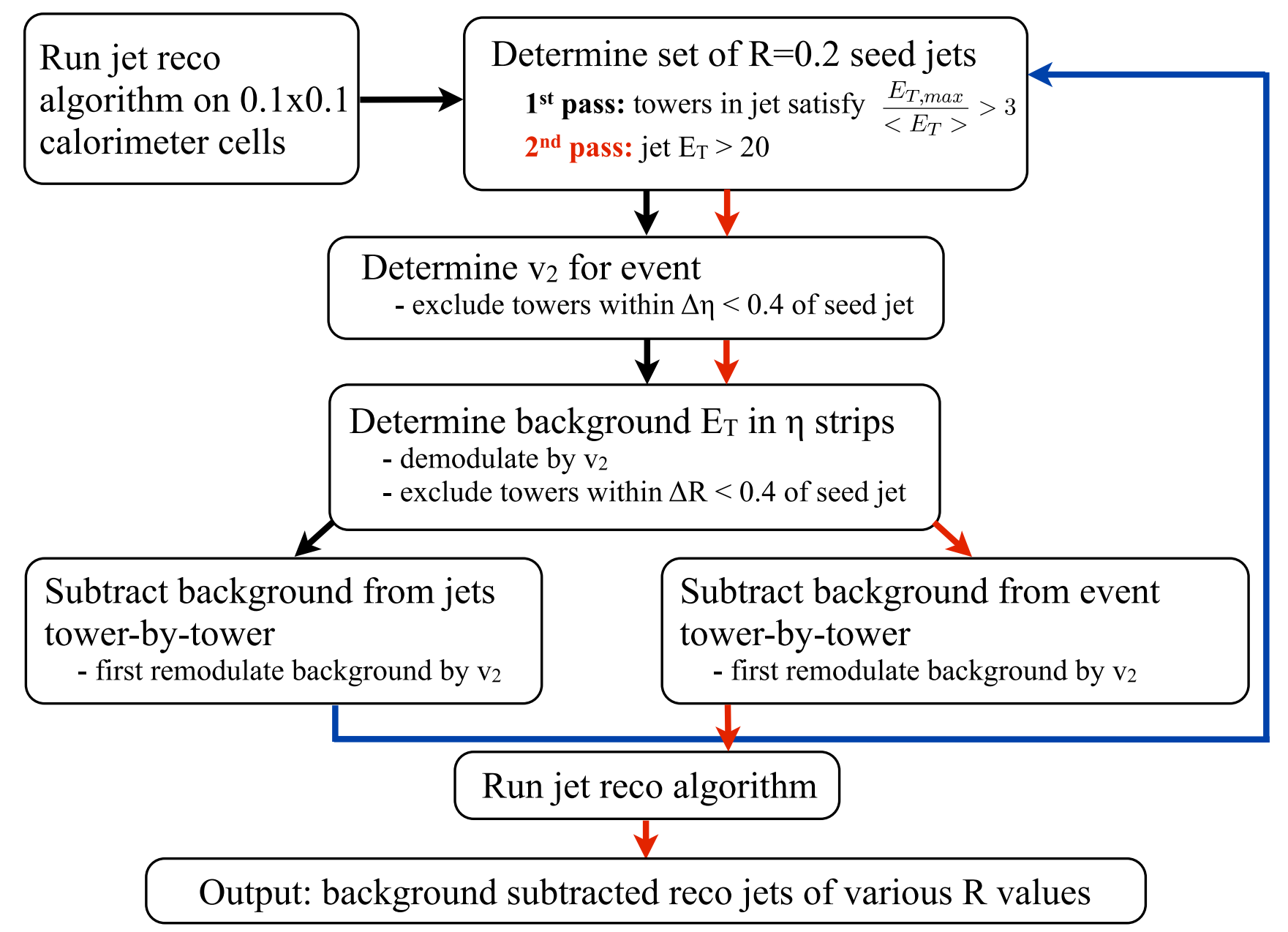

Figure $1 \quad$ CR10259 06Jul2012 


\section{Jet Event Display}

b=1.8 fermi, R=0.2, EVT\#9749, RUN\#404983

\section{True HIJING}

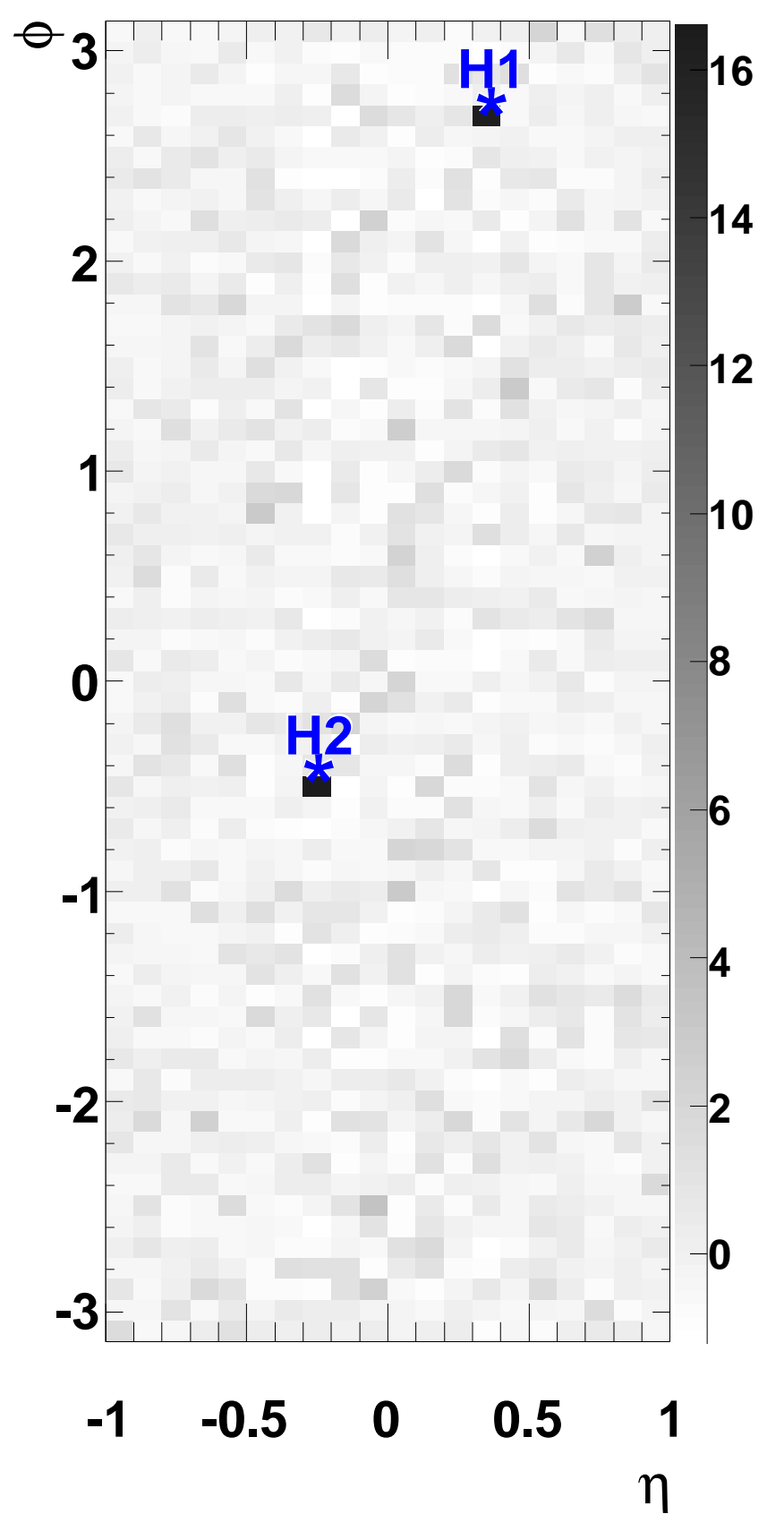

H1: $18 \mathrm{GeV} @<0.36,2.7>$

\section{Reconstructed}

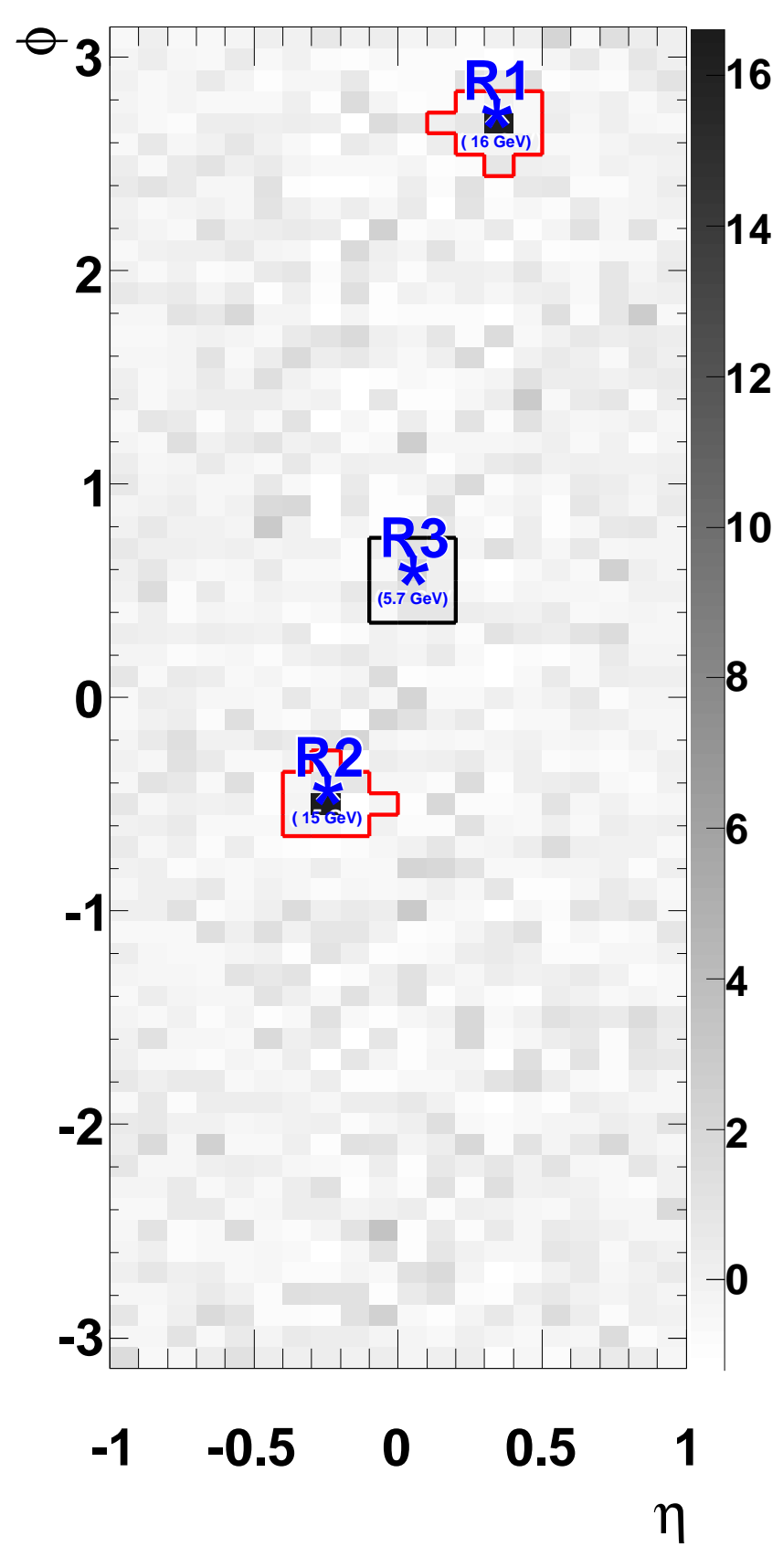

R1: $16 \mathrm{GeV} @<0.34,2.7>$; RMS=0.11

R2: $15 \mathrm{GeV} @<-0.24,-0.49>$; RMS=0.12

H2: $19 \mathrm{GeV} @<-0.25,-0.47>$ R3: $5.7 \mathrm{GeV} @<0.053,0.54>$; RMS=0.064 Figure 2 CR10259 06Jul2012 


\section{Jet Event Display}

\section{$b=2.4$ fermi, $R=0.4$, EVT\#1254, RUN\#400116}

\section{True HIJING}

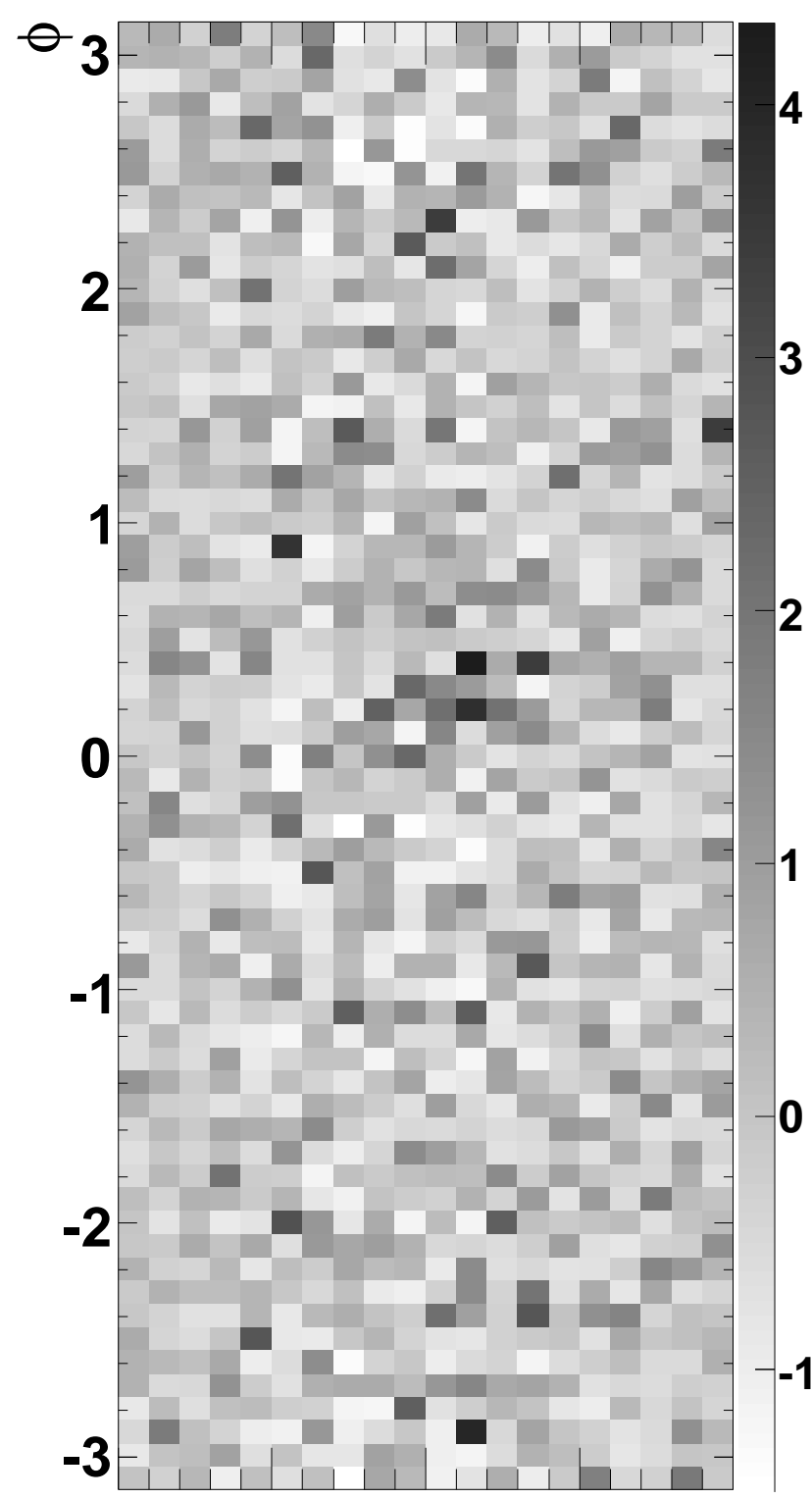

$\begin{array}{lllll}-1 & -0.5 & 0 & 0.5 & 1\end{array}$

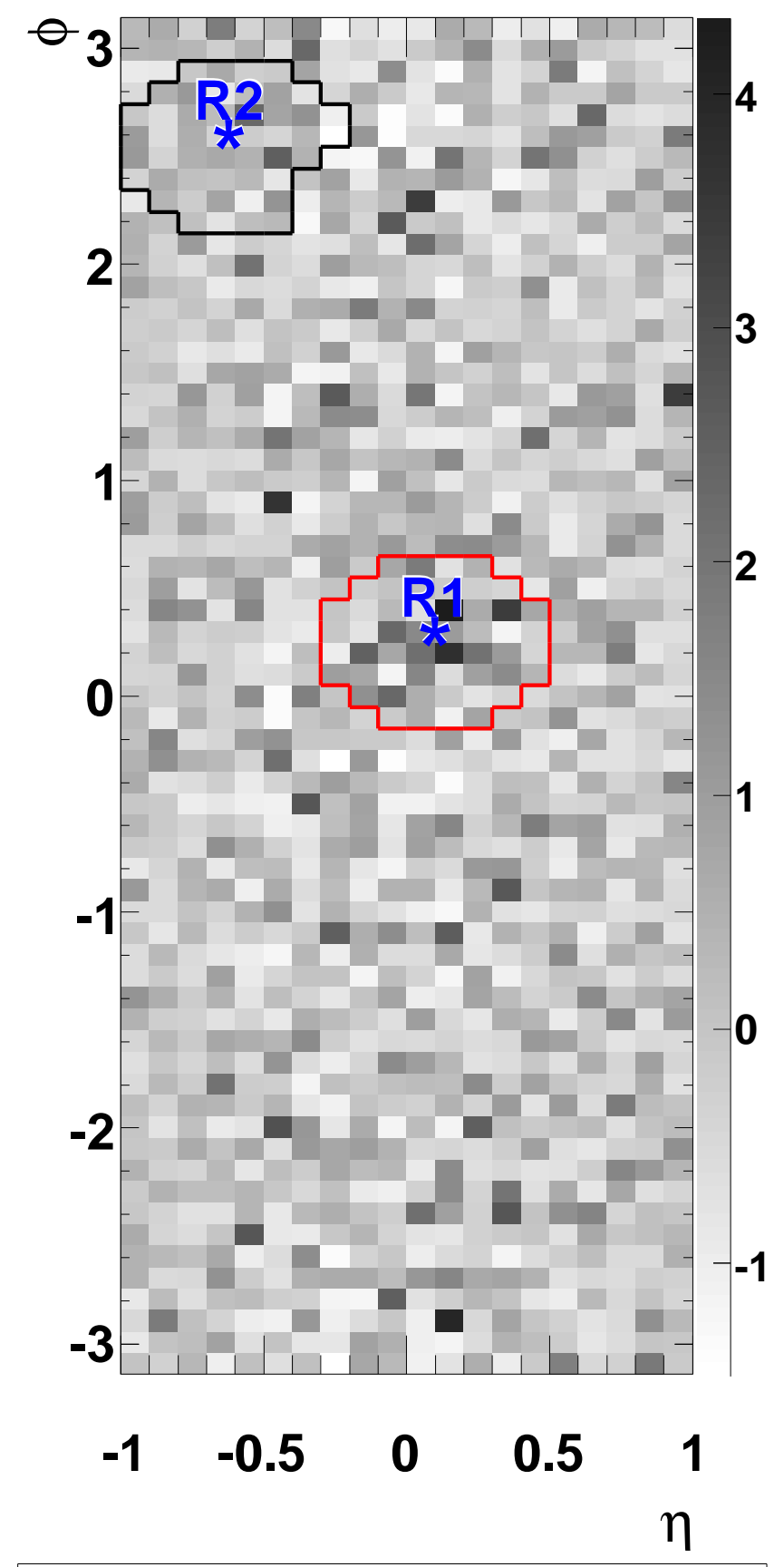

R1: $30 \mathrm{GeV} @<0.095,0.25>$; RMS=0.13

R2: $9.9 \mathrm{GeV} @<-0.63,2.6>$; RMS=0.072 


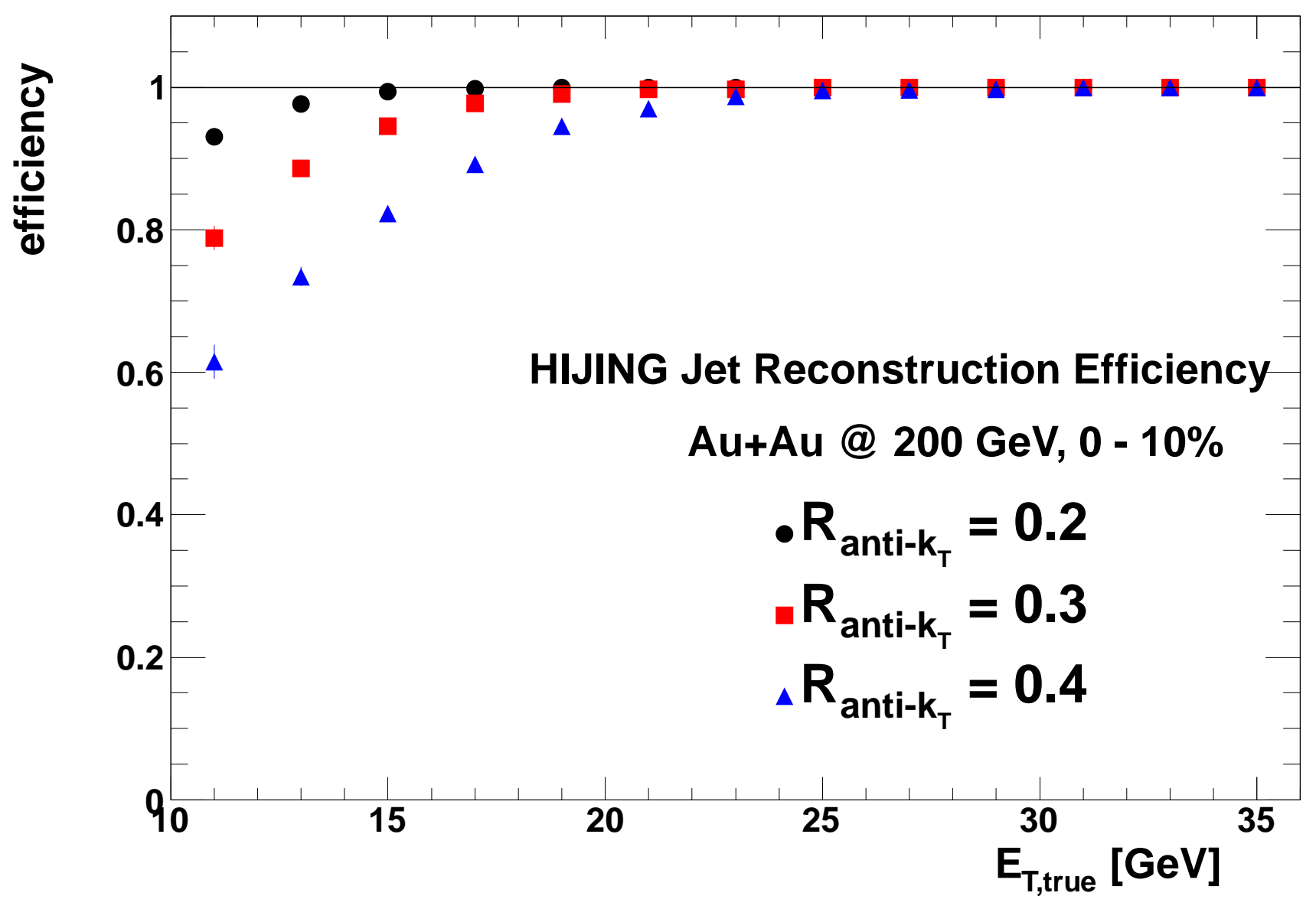

Figure $4 \quad$ CR10259 06Jul2012 


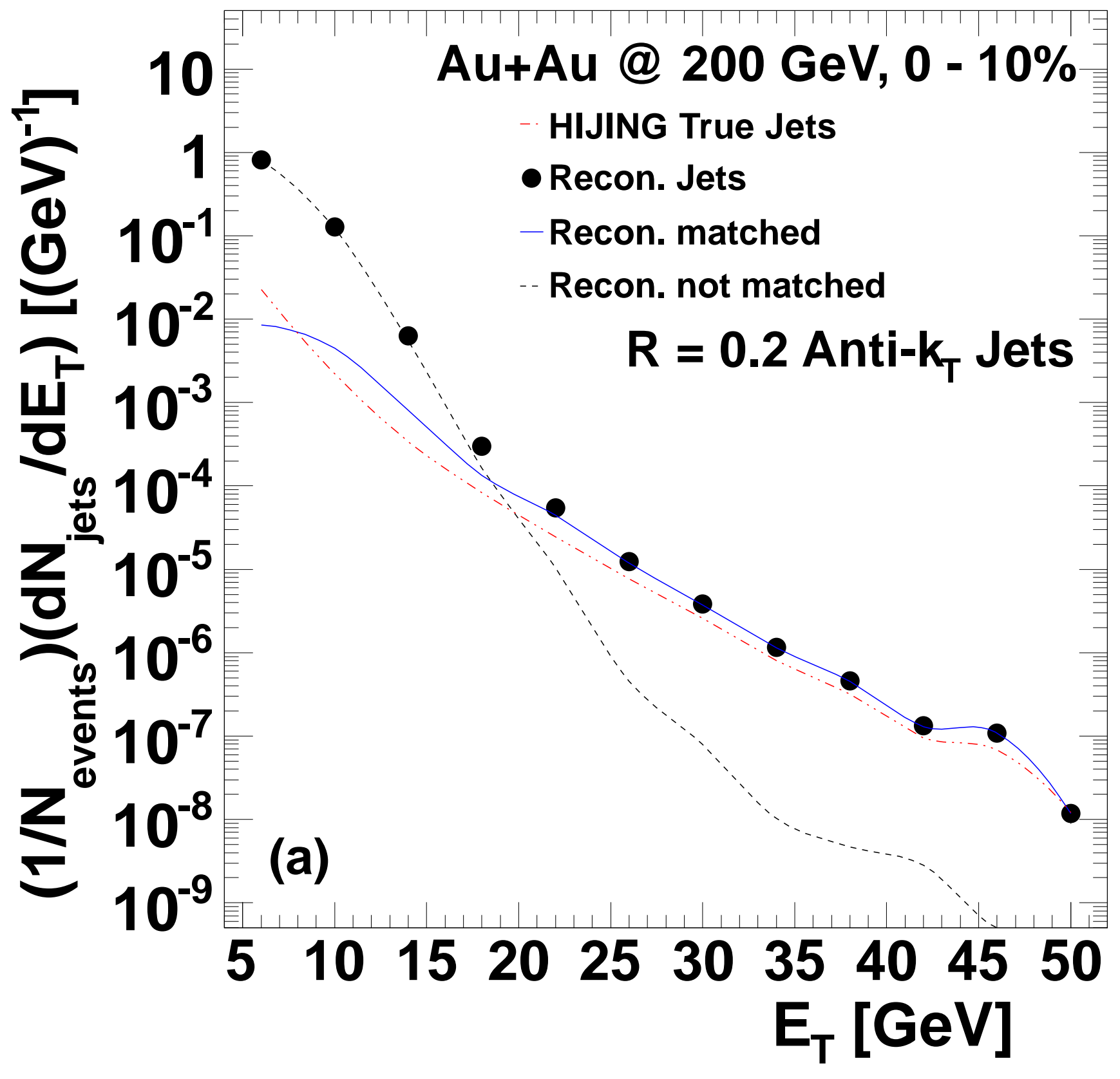

Figure 5a

CR10259 06Jul2012 


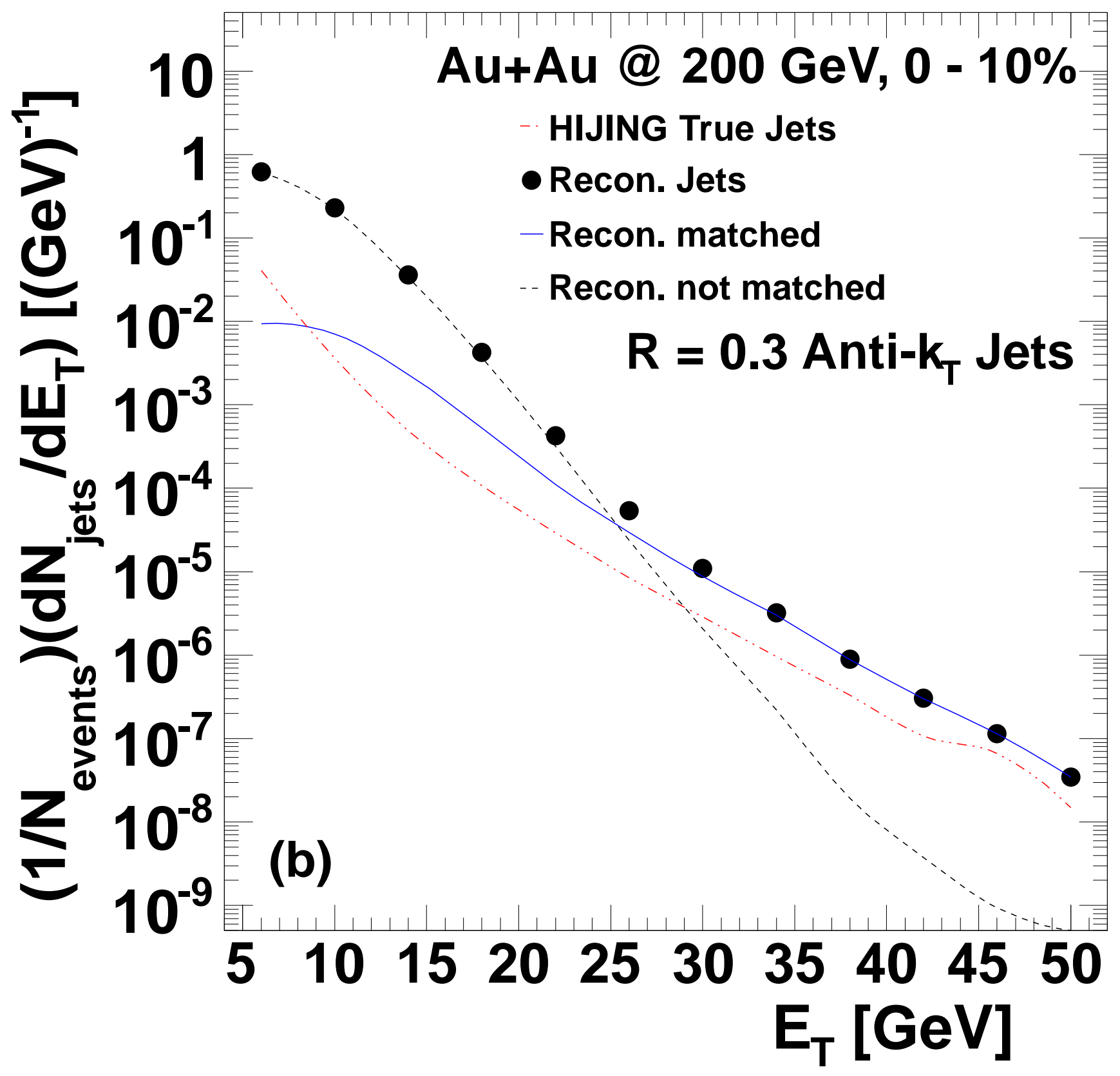

Figure $5 b$

CR10259 06Jul2012 


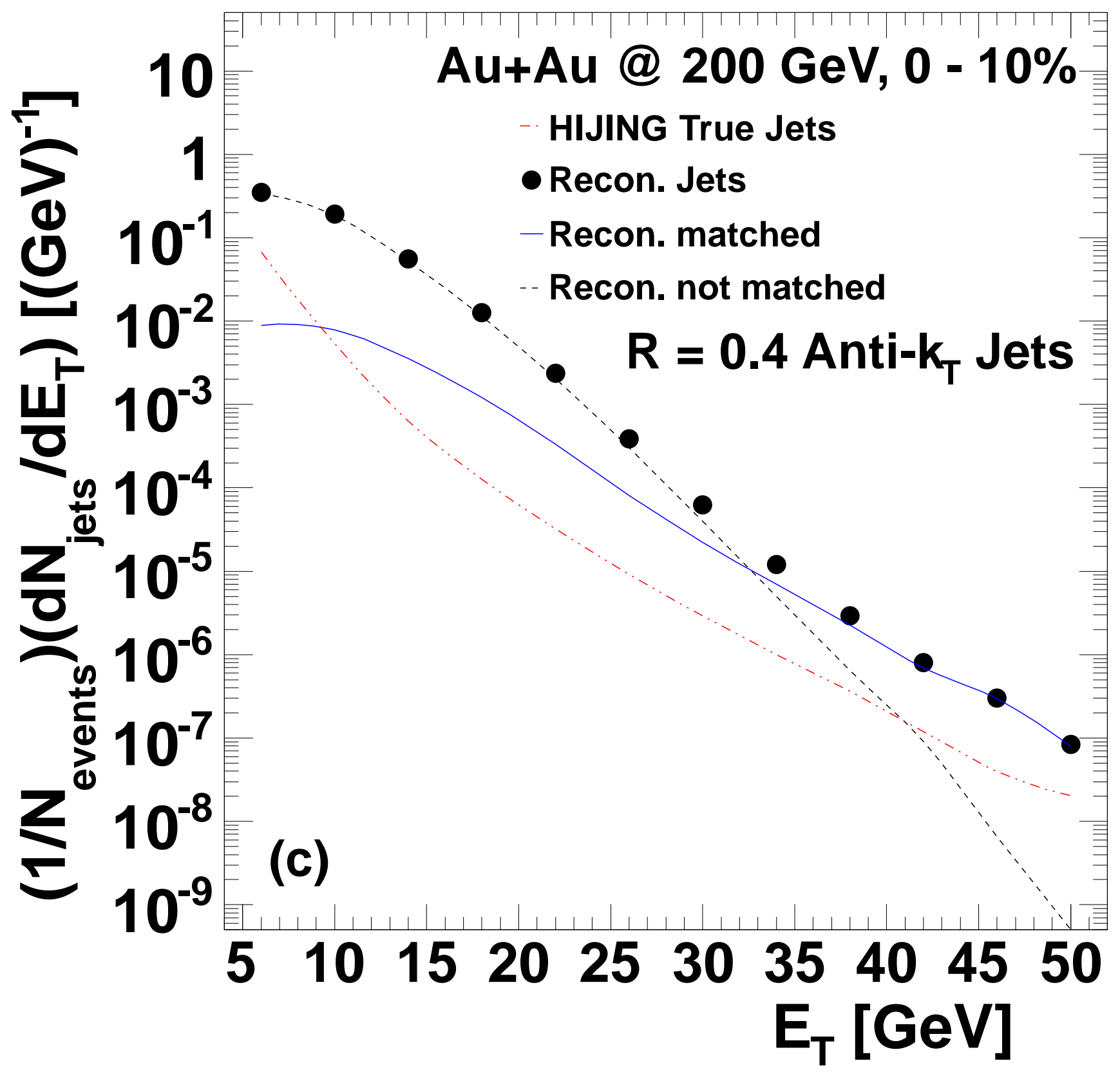

Figure 5c

CR10259 06Jul2012 


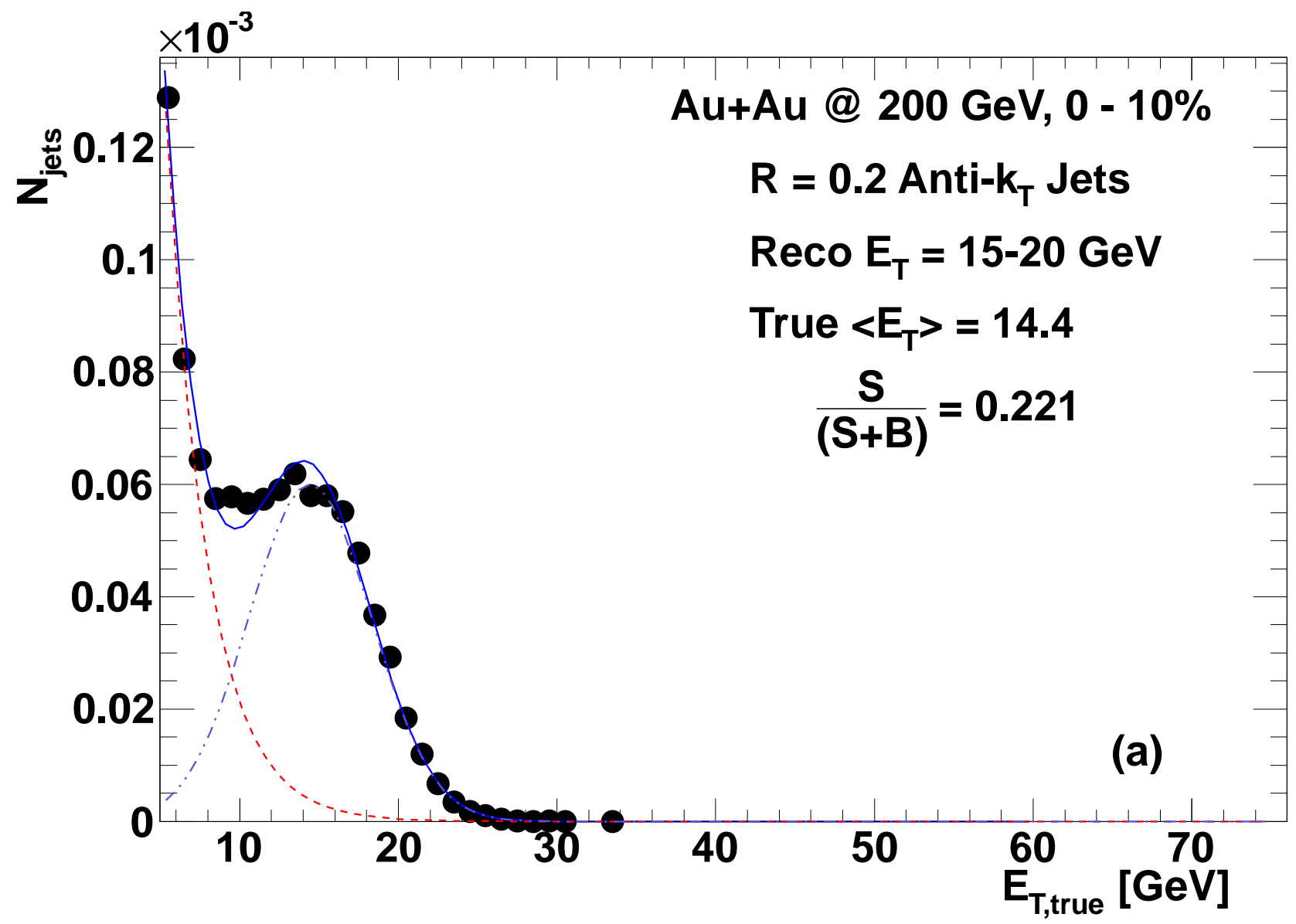

Figure 6a

CR10259 06Jul2012 


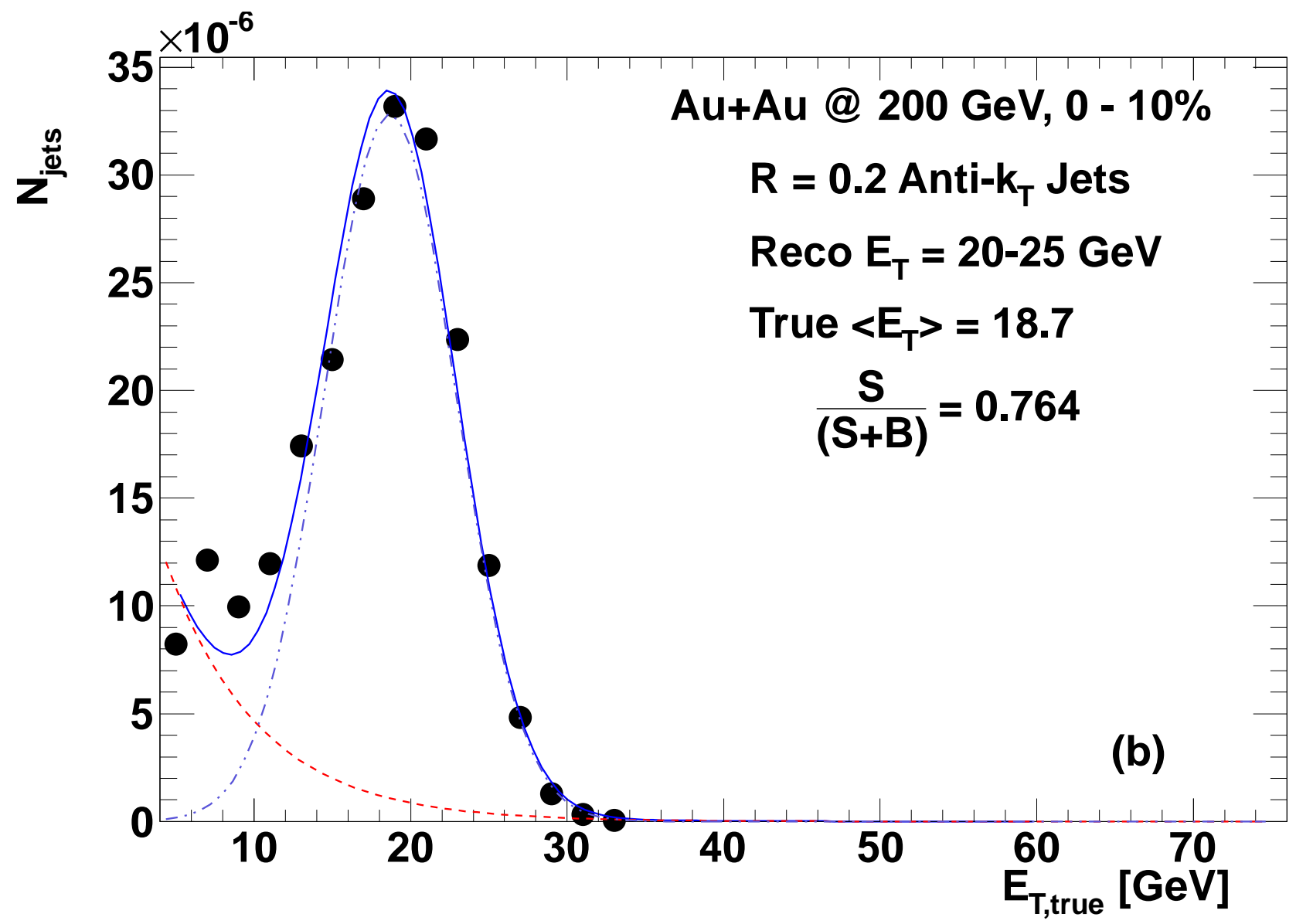

Figure 6b CR10259 06Jul2012 


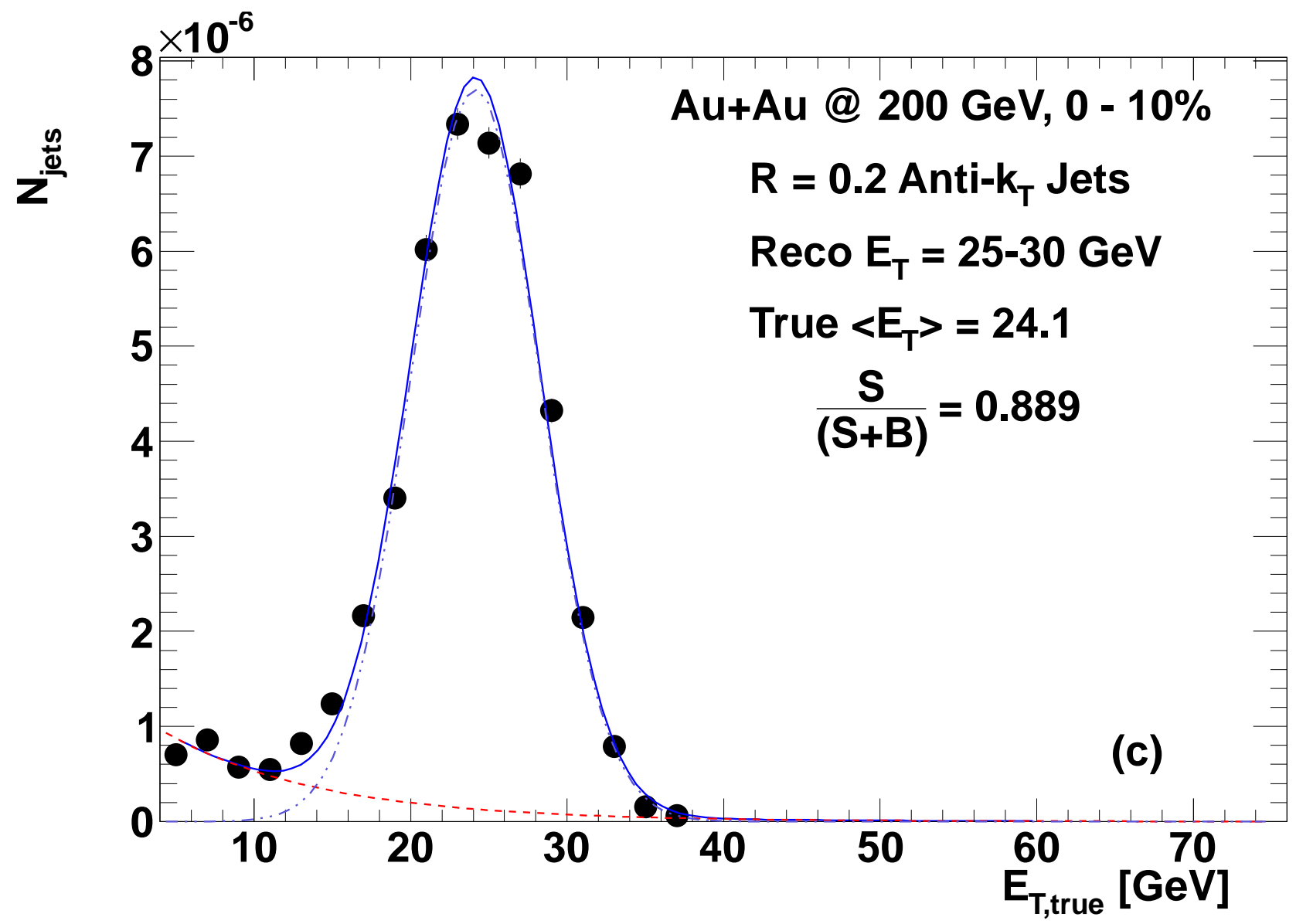

Figure 6c $\quad$ CR10259 06Jul2012 


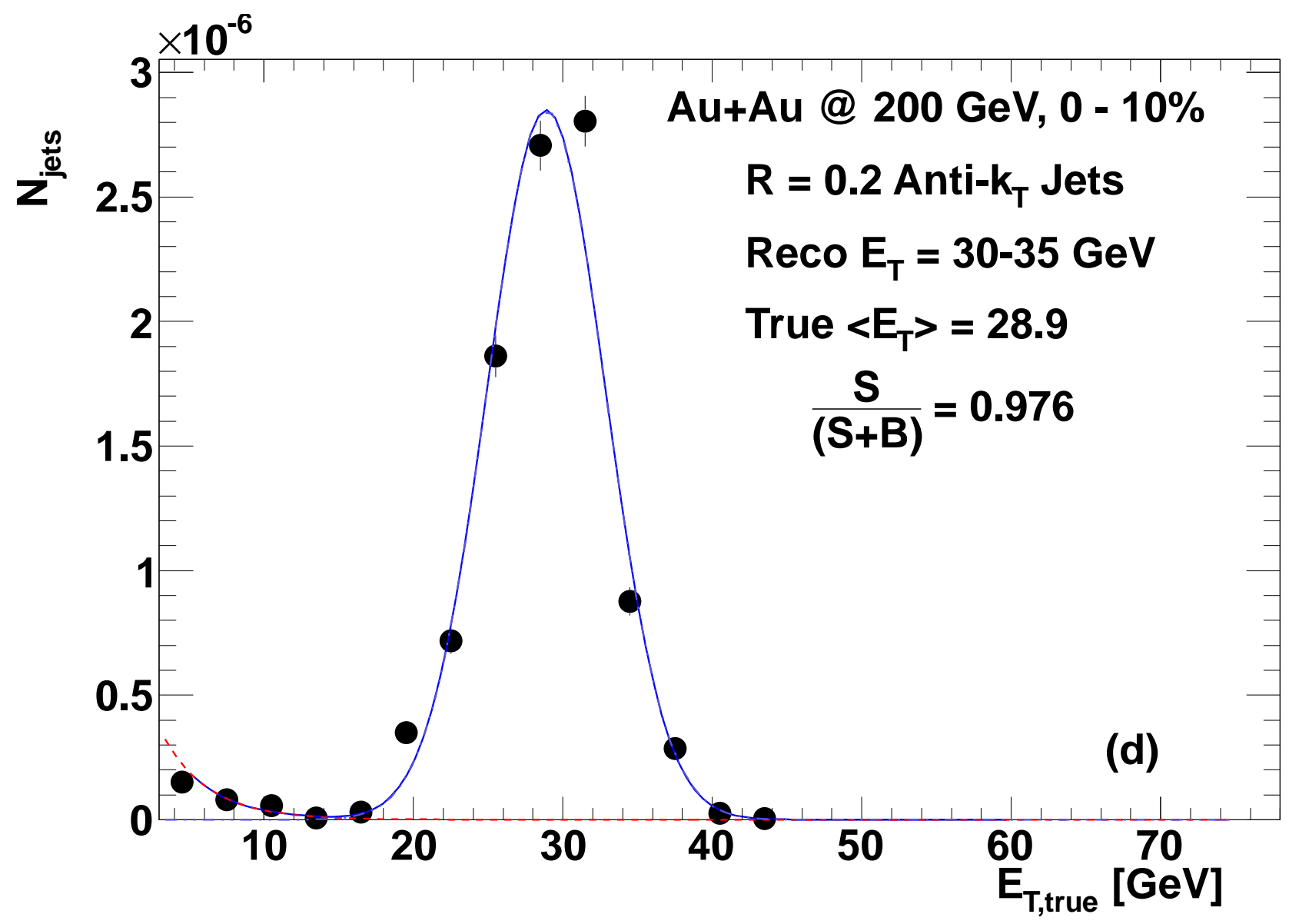

Figure 6d CR10259 06Jul2012 


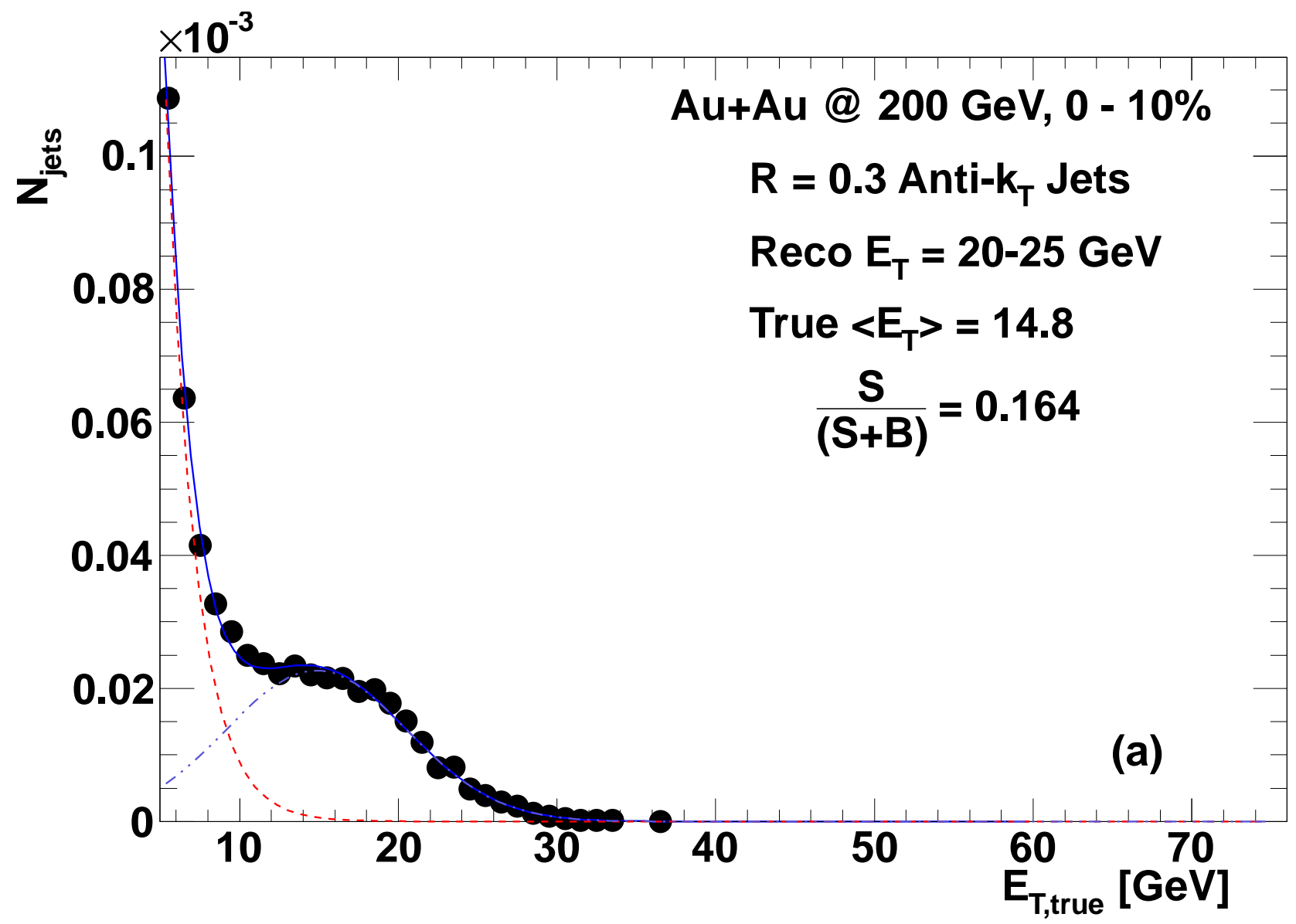

Figure 7a CR10259 06Jul2012 


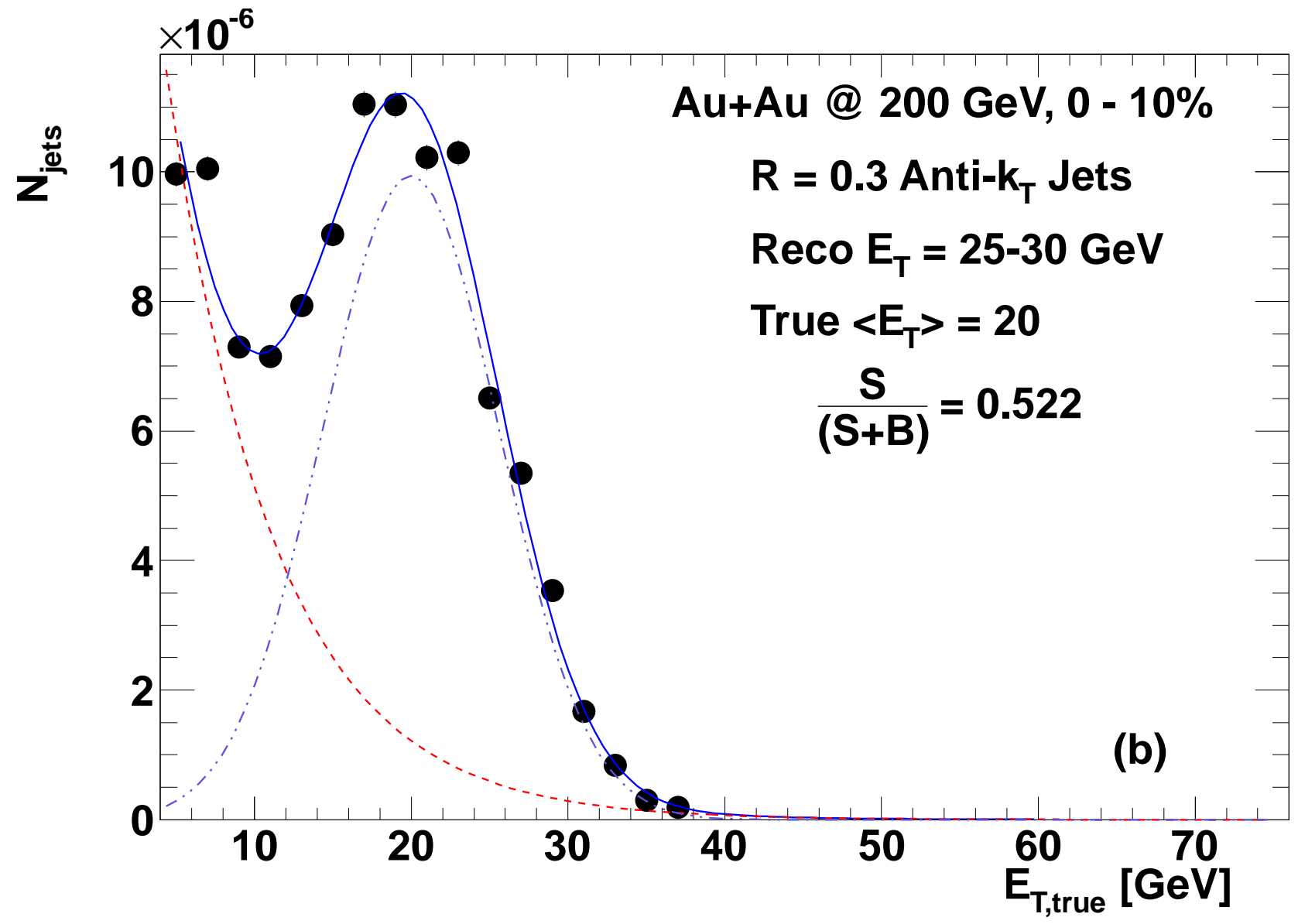

Figure 7b $\quad$ CR10259 06Jul2012 


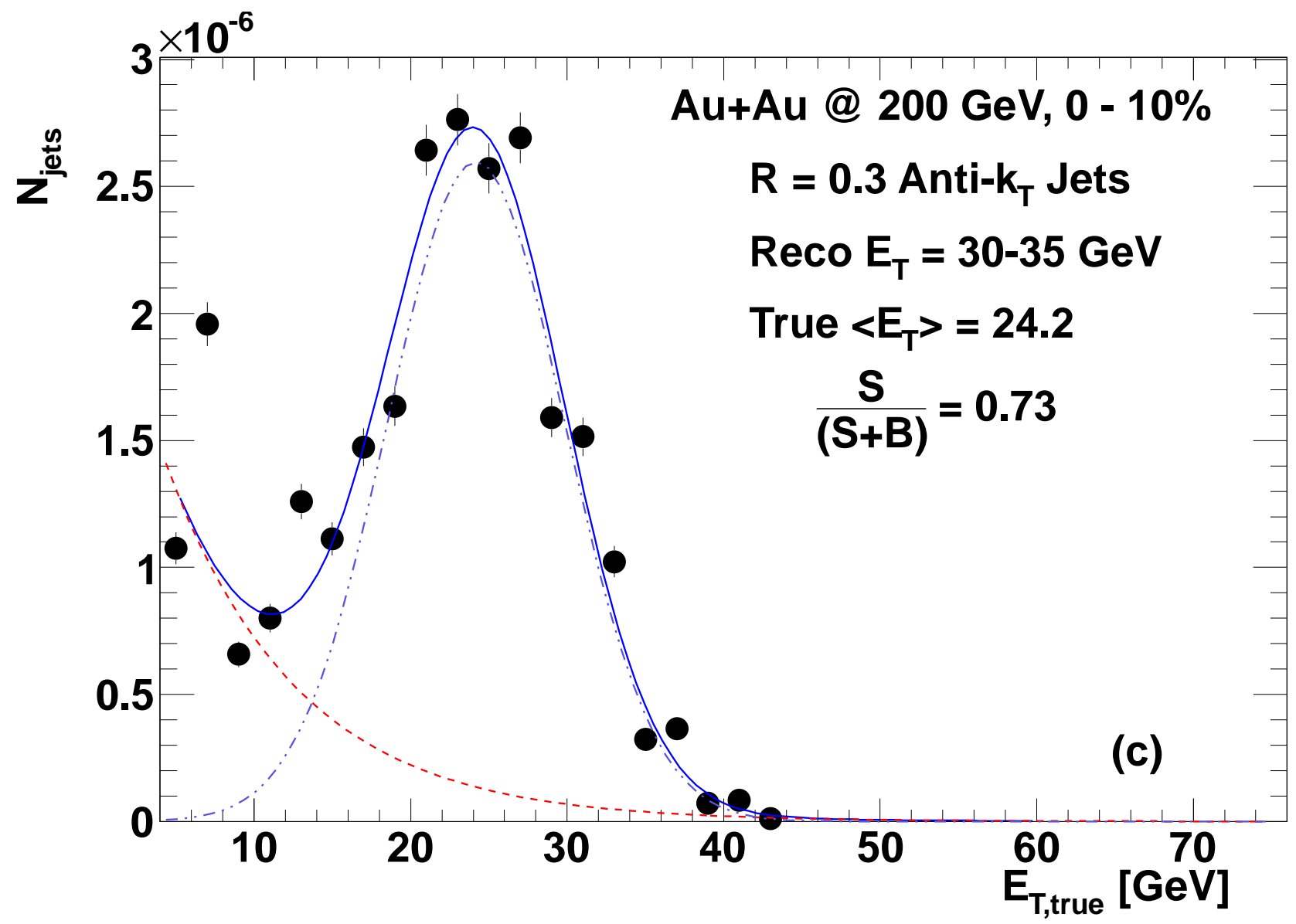

$\begin{array}{lll}\text { Figure 7c } & \text { CR10259 06Jul2012 }\end{array}$ 


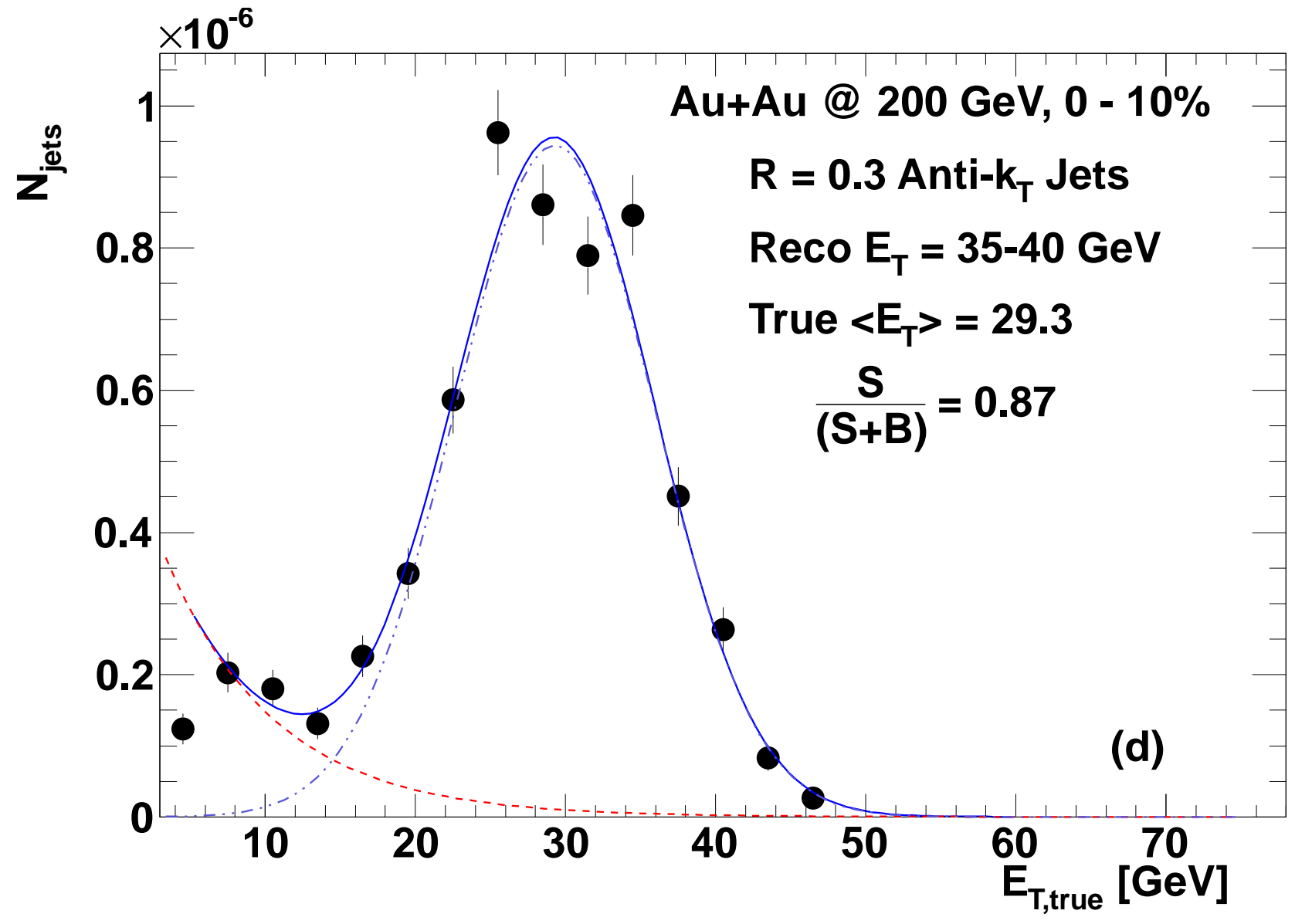

Figure 7d CR10259 06Jul2012 


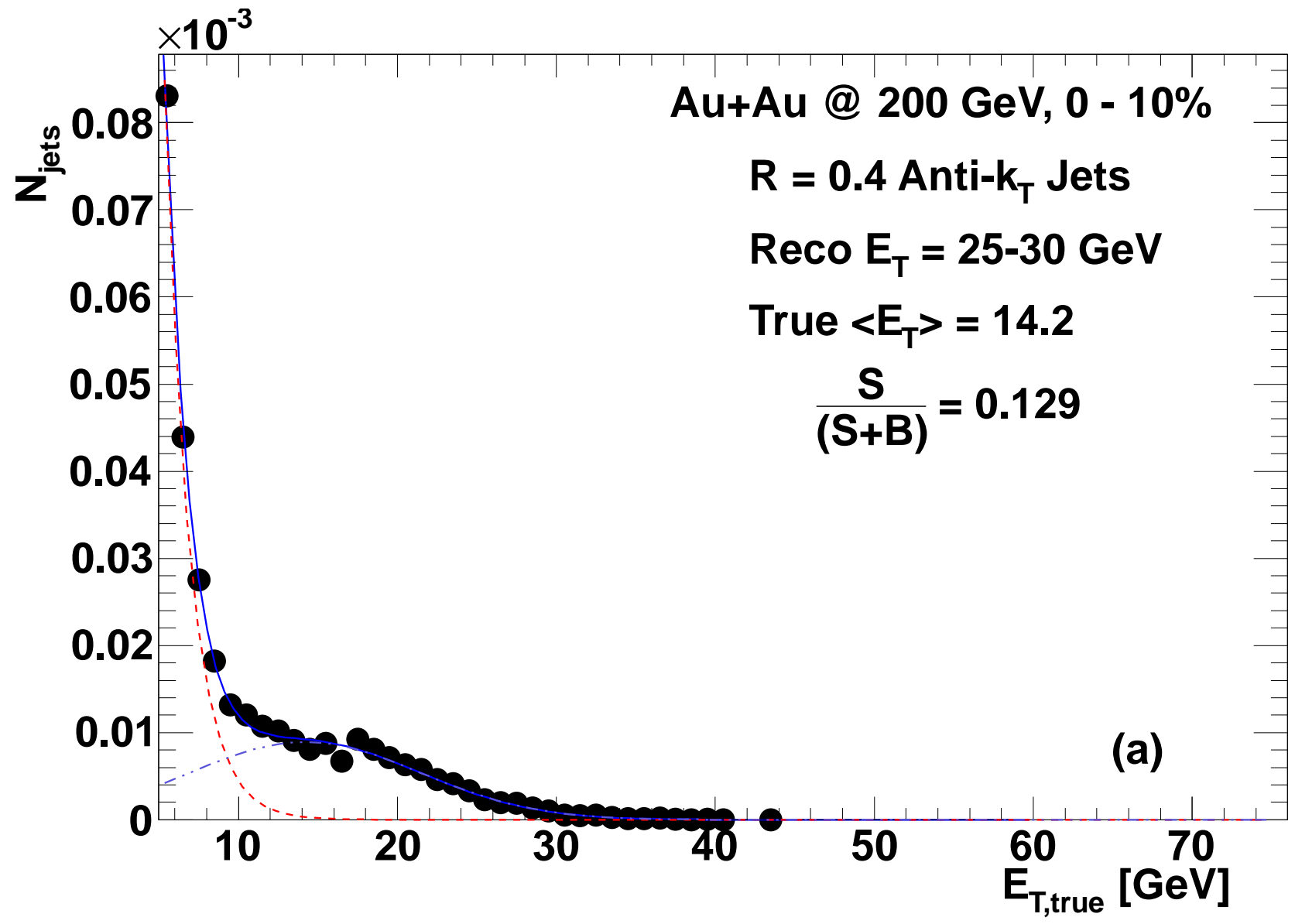

Figure 8a

CR10259 06Jul2012 


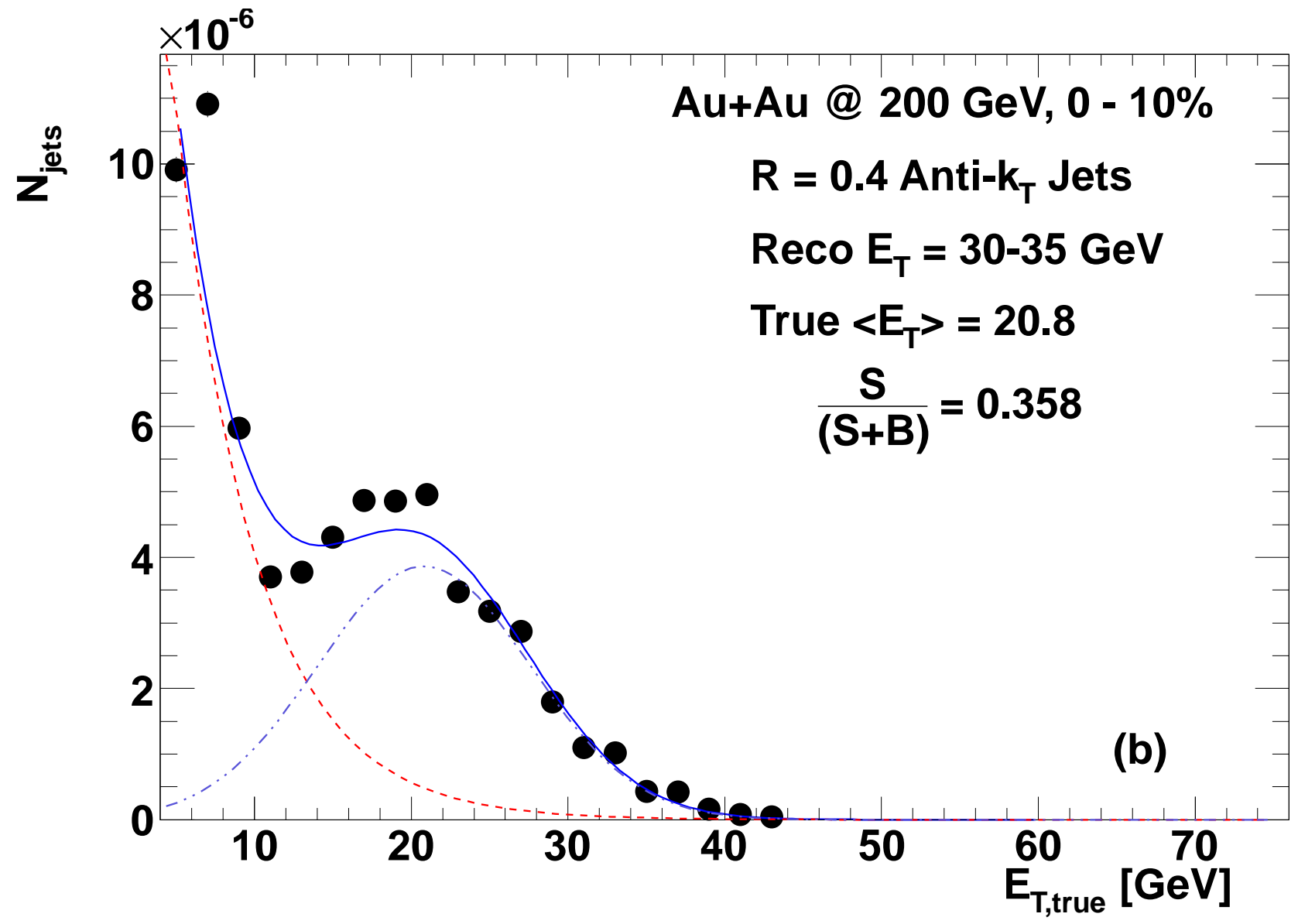

Figure 8b $\quad$ CR10259 06Jul2012 


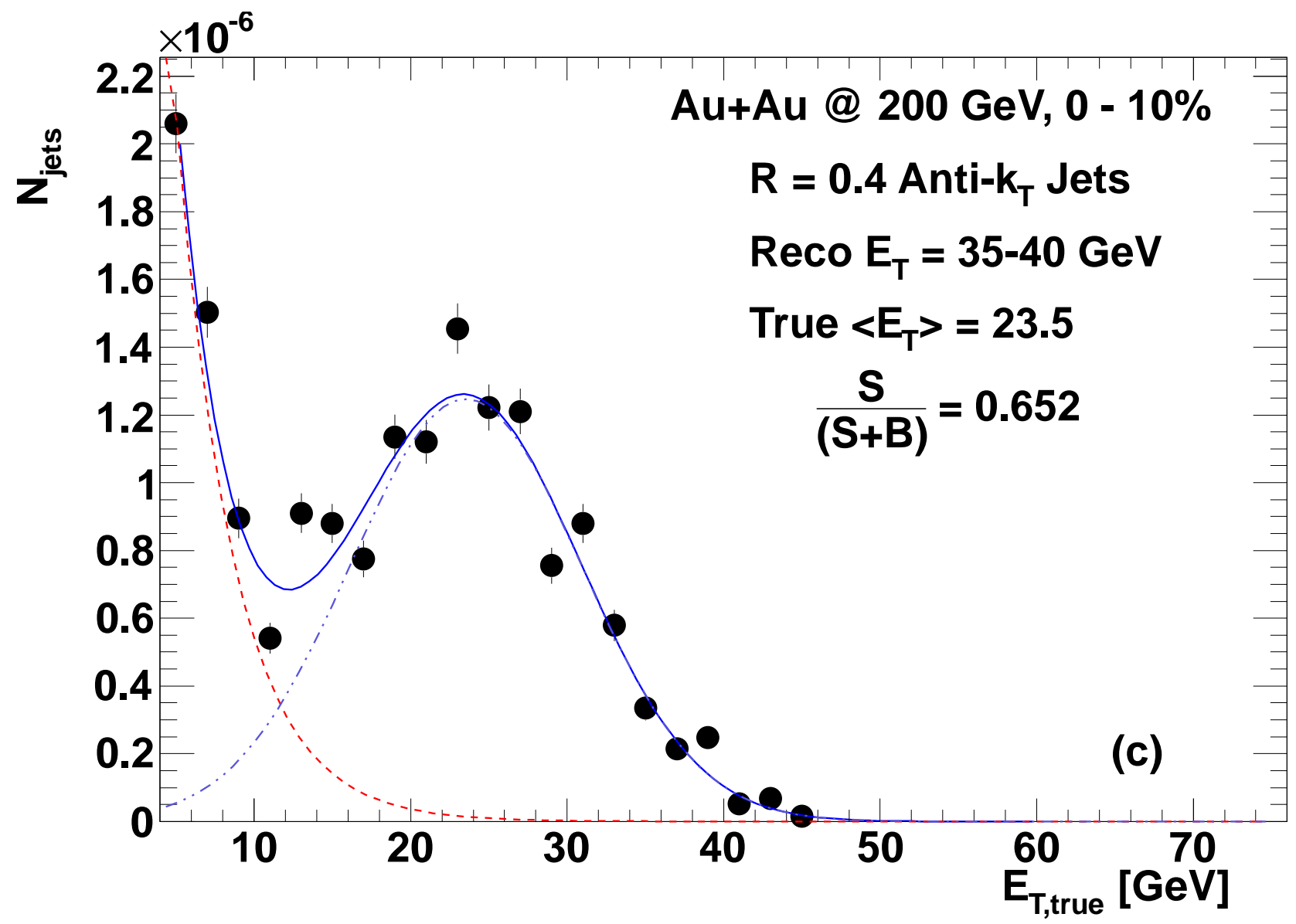

Figure 8c $\quad$ CR10259 06Jul2012 


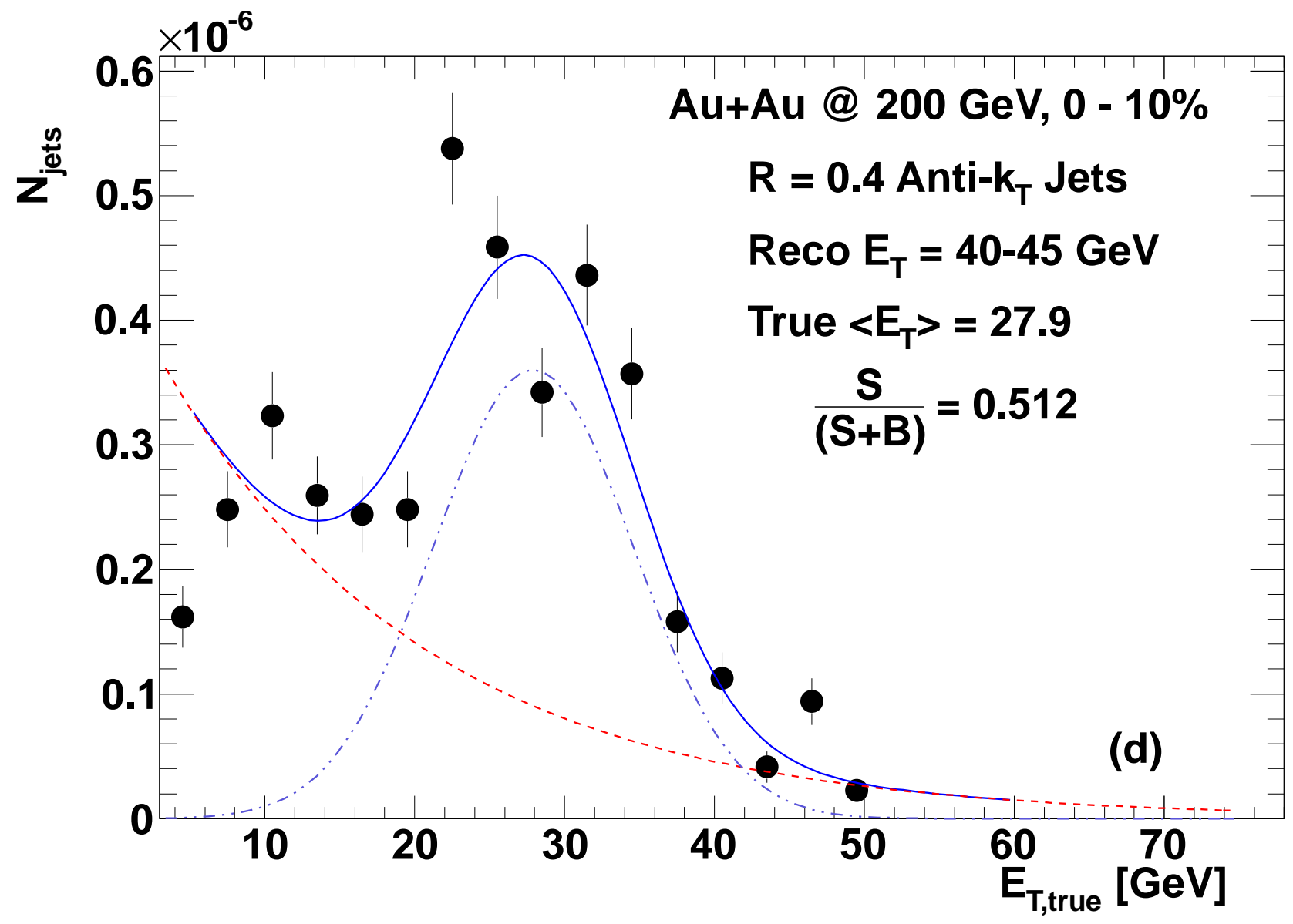

Figure 8d CR10259 06Jul2012 

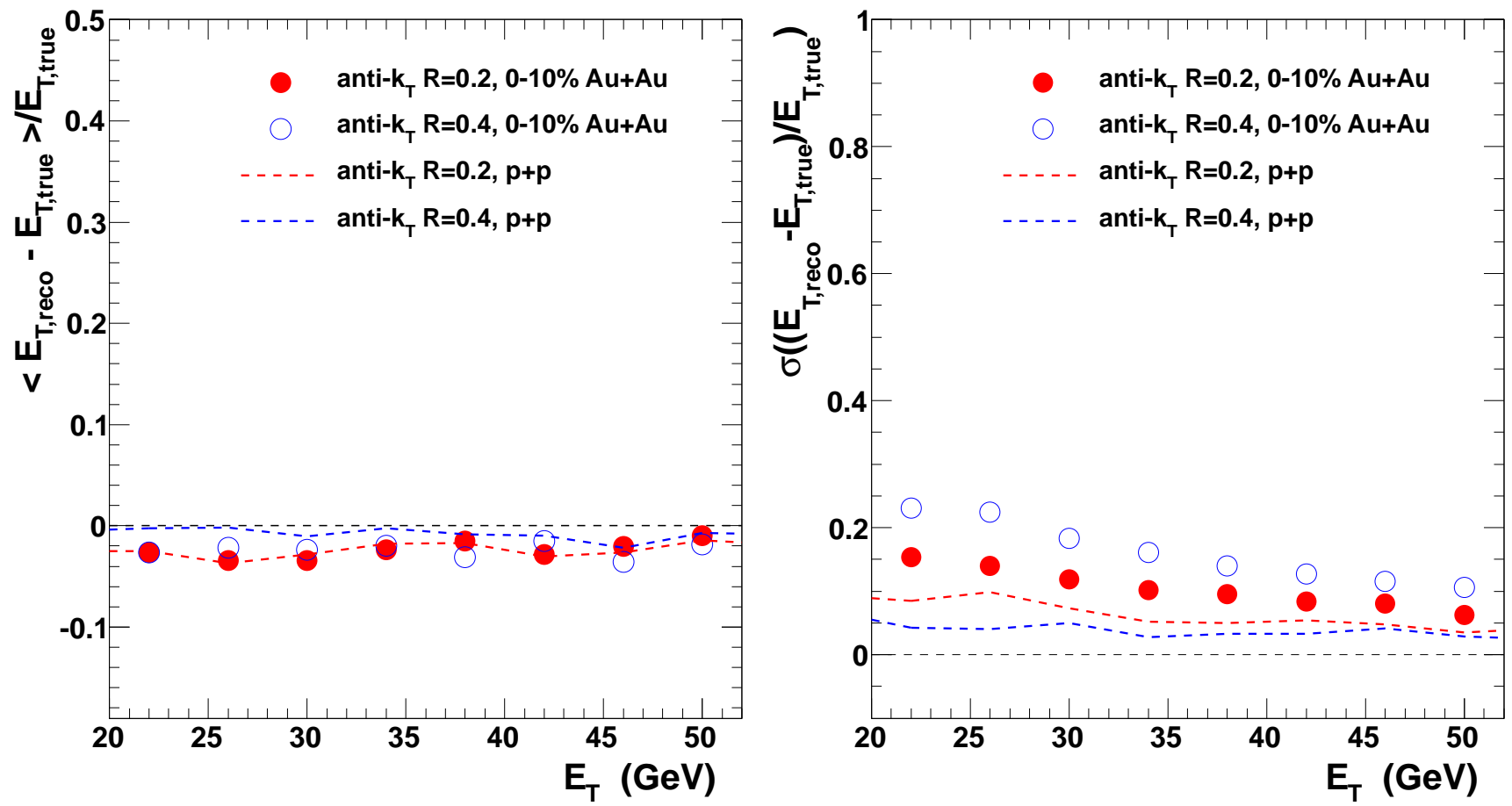